\title{
过渡金属硫属化合物层间异质结构的可控 制备和能源应用
}

史建平 ${ }^{1,2}$, 周协波 ${ }^{1,2}$, 张哲朋 ${ }^{2}$, 张艳锋 $1,2^{*}$

1. 北京大学工学院材料科学与工程系, 北京 100871 ;

2. 北京大学化学与分子工程学院纳米化学研究中心, 北京 100871

*联系人, E-mail: yanfengzhang@pku.edu.cn

2016-12-12 收稿, 2017-01-16 修回, 2017-01-20 接受, 2017-05-11 网络版发表

科技部重点研发计划(2016YFA0200103)、国家自然科学基金(51472008, 51290272, 21201012, 51121091, 51072004, 51201069)、北京市科学 技术委员会(Z151100003315013)和清华大学低维量子物理国家重点实验室开放基金(KF201601)资助

摘要随着石墨烯及其优异性质被发现以来，二维层状材料成为了材料科学领域研究的热点. 二维层状材料每 个片层内的原子通过化学键连接, 片层间以弱范德华力相互堆垛。这种几何结构使得二维层状材料在晶格不匹配 和生长方法不兼容的情况下，彼此之间仍然能够相互混合和匹配，从而衍生出很多范德华层间异质结构。这种异 质结构利用了不同堆垛材料迥异的物理和化学性质, 在电子、光电子器件、可再生能源储存和转化等领域得到了 广泛的应用。需要指出的是，大面积、大畴区、可控制备本征层间异质结构是实现其实际应用的首要条件. 本文总 结了基于过渡金属硫属化合物 $\left(\mathrm{MX}_{2}\right)$ 和石墨烯(graphene)层间异质结构的最新研究成果，重点描述了 $\mathrm{MX}_{2} /$ graphene 和 $\mathrm{MX}_{2} / \mathrm{MX}_{2}$ 层间异质结构的化学气相沉积 $(\mathrm{CVD})$ 可控制备、新奇物理性质探索以及这两类异质结构在能源领域 (电/光催化析氢反应)中的应用, 并讨论了所存在的问题和未来发展方向.

关键词石墨烯，过渡金属硫属化合物，层间异质结构，电催化析氢反应，光催化析氢反应

过渡金属硫属化合物是一类层状材料, 其化学 组成可表述为 $\mathrm{MX}_{2}$, 其中 $\mathrm{M}$ 代表过渡金属原子 $(\mathrm{Mo}$, $\mathrm{W}$ 等 $), \mathrm{X}$ 表示硫族原子 $\left(\mathrm{S}, \mathrm{Se}, \mathrm{Te}\right.$ 等)。单层 $\mathrm{MX}_{2}$ 是以 $\mathrm{X}-\mathrm{M}-\mathrm{X}$ 的形式通过共价化学键构筑而成, $\mathrm{MX}_{2}$ 的层与 层之间是非常弱的范德华相互作用 ${ }^{[1]}$. 单层 $\mathrm{MX}_{2}$ 具有 区别于块体材料的电学 ${ }^{[2]}$ 、光学 ${ }^{[3]}$ 、热学 ${ }^{[4]}$ 和力学 ${ }^{[5]}$ 等特性, 在高性能场效应晶体管 ${ }^{[6]}$ 、超导 ${ }^{[7]}$ 、非线性 光学 ${ }^{[8]}$ 和谷电子学 ${ }^{[9]}$ 等领域得到了广泛研究. 利用单 层 $\mathrm{MX}_{2}$ 直接带隙的能带结构 ${ }^{[1]}$ 和石 墨烯 (graphene) 超 高载流子迁移率 ${ }^{[10]}$ 构筑而成的 $\mathrm{MX}_{2} /$ graphene层间异 质结构, 近年来在电子 ${ }^{[2]}$ 、光电子 ${ }^{[11]}$ 和电催化析氢反 应 $^{[12]}$ 等领域得到了广泛应用. 除 $\mathrm{MX}_{2} /$ graphene层间
异质结构以外, 具有不同带隙宽度的单层 $\mathrm{MX}_{2}$ 堆垛 而成的 $\mathrm{MX}_{2} / \mathrm{MX}_{2}$ 层间异质结构也引起了人们的极大 关注 ${ }^{[13,14]}$. 理论计算表明, $\mathrm{MoS}_{2} / \mathrm{WS}_{2}$ 层间异质结构 是一种 II 型半导体, 导带和价带分别位于 $\mathrm{MoS}_{2}$ 和 $\mathrm{WS}_{2}$ 上, 这种 II 型能带结构实现了电子-空穴对的自 发分离, 在超快电荷传输和光电探测等领域具有潜 在的应用价值 ${ }^{[13 \sim 15]}$.

需要指出的是, $\mathrm{MX}_{2} /$ graphene和 $\mathrm{MX}_{2} / \mathrm{MX}_{2}$ 层间异 质结构通常采用机械剥离/层层堆垛以及液相化学反 应的方法获得. 机械剥离/层层堆垛构筑的 $\mathrm{MX}_{2} / \mathrm{gra}-$ phene和 $\mathrm{MX}_{2} / \mathrm{MX}_{2}$ 层间异质结构具有很高的结晶质 量, 适用于基础结构表征和光电原型器件构建. 但 
是, 这种制备方法很难实现异质结构的批量生产以 及畴区尺寸、层厚和层间扭转角度的精确控制. 液相 化学反应制备的 $\mathrm{MX}_{2} /$ graphene层间异质结构, 其结 晶质量相比机械剥离法有明显降低. 同时溶液相的 引人有可能污染异质结构的表/界面, 不利于其在电/ 光学器件上的实际应用.

化学气相沉积 (chemical vapor deposition, CVD) 法是一种能够制备高质量、大面积、层厚可控纳米材 料的方法. 目前这种方法被广泛地应用于石墨 烯 ${ }^{[16,17]}$ 、氮化硼 ${ }^{[18,19]}$ 和 $\mathrm{MX}_{2}{ }^{[20 ~ 27]}$ 等二维层状材料的制 备. 2012 年美国麻省理工学院Kong 课题组 ${ }^{[28]}$ 以 $\left(\mathrm{NH}_{4}\right)_{2} \mathrm{MoS}_{4}$ 为前驱体, 在铜箔上 $\mathrm{CVD}$ 生长的石墨烯 上制备了 $\mathrm{MoS}_{2}$. 然而, 这种方法获得的 $\mathrm{MoS}_{2}$ 畴区尺 寸小 $(<100 \mathrm{~nm})$ 、层厚不可控(单层和多层共存). 随后 美国海军实验室Jonker课题组 ${ }^{[29]}$ 将铜箔上CVD生长 的石墨烯转移到 $\mathrm{SiO}_{2} / \mathrm{Si}$ 衬底上, 以 $\mathrm{MoO}_{3}$ 和 $\mathrm{S}$ 粉为前 驱体, 利用常压CVD的方法在 graphene $/ \mathrm{SiO}_{2} / \mathrm{Si}$ 上制 备了单层 $\mathrm{MoS}_{2}$ 薄膜. 但是, 石墨烯转移过程的引人, 使得所制备 $\mathrm{MoS}_{2} /$ graphene层间异质结构的界面受到 了有机物污染, 无法获得 $\mathrm{MoS}_{2} /$ graphene本征的光电 特性. 此外, $\mathrm{CVD}$ 的方法也被广泛应用于 $\mathrm{MX}_{2} / \mathrm{MX}_{2}$ 层 间异质结构的可控制备. 美国莱斯大学Ajayan课题 组 ${ }^{[30]}$ 利用常压 $C V D$ 的方法, 以 $\mathrm{MoO}_{3}, \mathrm{~W}$ 和 $\mathrm{S}$ 粉为前驱 体, 在 $\mathrm{SiO}_{2} / \mathrm{Si}$ 衬底上制备了 $\mathrm{WS}_{2} / \mathrm{MoS}_{2}$ 层间异质结构. 然而, 由于成长气氛中 $\mathrm{Mo}$ 和 $\mathrm{W}$ 元素共存, 所制备 $\mathrm{WS}_{2} / \mathrm{MoS}_{2}$ 层间异质结构发生了 $\mathrm{Mo}$ 和 W两种元素的 复混和掺杂, 无法获得本征 $\mathrm{WS}_{2} / \mathrm{MoS}_{2}$ 层间异质结构. 随后, 清华大学焦丽颖课题组 ${ }^{[31]}$ 以 $\mathrm{WO}_{3-x} / \mathrm{MoO}_{3-x}$ 核 壳纳米线作为前驱体, 实现了 $\mathrm{MoS}_{2}$ 和 $\mathrm{WS}_{2}$ 的顺序生 长, 进而获得了本征 $\mathrm{WS}_{2} / \mathrm{MoS}_{2}$ 层间异质结构.

本课题组 ${ }^{[32,33]}$ 利用两步 CVD的方法实现了金管 $(\mathrm{Au})$ 上 $\mathrm{MoS}_{2} / \mathrm{graphene}$ 层间异质结构的可控制备. 由 于制备中没有任何转移过程的引人, 获得的 $\mathrm{MoS}_{2}$ / graphene层间异质结构具有干净和本征的界面. 值得 一提的是, 利用这种方法获得的 $\mathrm{MoS}_{2}$ 均是单层厚度. 通过调控 graphene/ $\mathrm{Au}$ 和前驱体 $\left(\mathrm{MoO}_{3}\right)$ 之间的距离, 实现了 graphene/ $\mathrm{Au}$ 上 $\mathrm{MoS}_{2}$ 的覆盖度从亚单层到满层 的调节. 利用低温扫描隧道显微镜/谱(scanning tunneling microscopy and spectroscopy, STM/STS)实现了 $\mathrm{MoS}_{2} /$ graphene原子尺度形貌和本征局域电子结构的 直接观测. 结合STS和光致发光谱(photoluminescence, $\mathrm{PL}$ ), 获得了 $\mathrm{MoS}_{2} /$ graphene层间异质结构的激子结合
能. 考虑到氢离子在 $\mathrm{MoS}_{2} /$ graphene活性位点具有较 低的吸附势垒, 将 $\mathrm{MoS}_{2} / \mathrm{graphene} / \mathrm{Au}$ 直接用于电催化 析氢反应, 并实现了高效的产氢效果 ${ }^{[34]}$. 此外, 选用 两步 CVD的生长方法, 通过生长温度和生长顺序的调 控, 在 $\mathrm{Au}$ 䇴上选择性地构筑了 $\mathrm{MoS}_{2} / \mathrm{WS}_{2}$ 和 $\mathrm{WS}_{2} / \mathrm{MoS}_{2}$ 层间异质结构. 两步 CVD方法的选择有效避免了不 同过渡金属元素 (Mo和 W) 之间的互混和掺杂，直接 获得了更加本征和高质量的异质结构 ${ }^{[35]}$. 需要指出 的是, 在 $\mathrm{MoS}_{2} / \mathrm{WS}_{2}$ 异质结构中, A-A堆垛形式占主 导, 而 $\mathrm{WS}_{2} / \mathrm{MoS}_{2}$ 异质结构中, A-B堆垛形式占优. 更 有趣的是, 这两种不同堆垛顺序的异质结构在光催 化析氢反应中显示出了截然相反的效果. 光照下 $\mathrm{MoS}_{2} / \mathrm{WS}_{2}$ 的析氢效率明显增强, 而 $\mathrm{WS}_{2} / \mathrm{MoS}_{2}$ 的析氢 效果则减弱. 本文将以这些研究成果为主线, 系统地 阐述 $\mathrm{MX}_{2} /$ graphene和 $\mathrm{MX}_{2} / \mathrm{MX}_{2}$ 层间异质结构的可控 制备、精密表征及在电/光催化析氢反应中的应用, 并 对该领域的未来发展趋势以及面临的挑战进行简要 的总结和展望.

\section{$1 \mathrm{MX}_{2} /$ graphene层间异质结构的应用和制 备方法}

顺序堆垛单层 $\mathrm{MX}_{2}$ 和石墨烯所构筑的层间异质

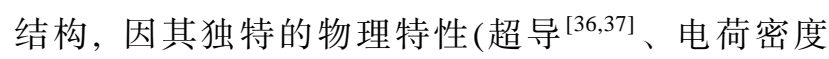

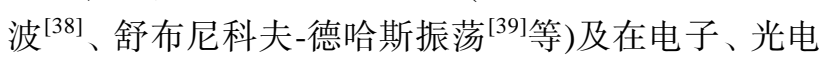
子器件、可再生能源储存和转化等领域多元的应用前

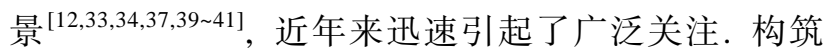
高质量本征 $\mathrm{MX}_{2} /$ graphene层间异质结构是实现新奇 物理性质研究和应用探索的前提.

理论计算表明, 氢离子在 $\mathrm{MoS}_{2} /$ graphene层间异 质结构 $(0.11 \mathrm{eV})$ 活性位点的吸附势垒相比单一 $\mathrm{MoS}_{2}$ $(0.17 \mathrm{eV})$ 明显降低 ${ }^{[42]}$. 因此, $\mathrm{MoS}_{2} / \mathrm{graphene}$ 有望实现 更加高效的电催化产氢. 美国斯坦福大学Dai课题 组 ${ }^{[43]}$ 利用溶剂热合成法, 以 $\left(\mathrm{NH}_{4}\right)_{2} \mathrm{MoS}_{4}$ 为前驱体, 在 还原氧化石墨烯(reduced graphene oxide, rGO)上制备 了 $\mathrm{MoS}_{2}$ 纳米粒子. $\mathrm{MoS}_{2}$ 纳米粒子丰富的催化活性位 点以及 $\mathrm{MoS}_{2}$ 与 $\mathrm{rGO}$ 之间强电子耦合, 使得 $\mathrm{MoS}_{2} / \mathrm{rGO}$ 表现出了极强的电催化析氢特性, 开启电压为 $0.1 \mathrm{~V}$, 塔菲尔斜率为 $41 \mathrm{mV} / \mathrm{dec}$ (图 1(a)). 随后, 美国罗格斯 大学 Chhowalla课题组 ${ }^{[12]}$, 以 $\mathrm{WCl}_{6}$ 和 $\mathrm{C}_{2} \mathrm{H}_{5} \mathrm{NS}$ 作为前 驱体通过液相化学反应的方法在 $\mathrm{rGO}$ 上制备了 $\mathrm{WS}_{2}$ 的 纳米片, 塔菲尔斜率为 $58 \mathrm{mV} \mathrm{dec}{ }^{-1}$.

考虑到单层 $\mathrm{MX}_{2}$ 的直接带隙能带结构和石墨烯 
超快的电子传输性能, $\mathrm{MX}_{2} / \mathrm{graphene}$ 层间异质结构在 电子和光电子器件上具有广阔的应用前景. 英国曼 彻斯特大学Novoselov课题组 ${ }^{[44]}$ 以机械剥离单层 $\mathrm{WS}_{2}$ 和石墨烯构筑了 graphene/ $\mathrm{WS}_{2} /$ graphene光伏器件. $\mathrm{WS}_{2}$ 电子态密度中的范霍夫奇异点 (Van Hove singularities)增强了该器件的光/物相互作用, 进而导致了 更强的光吸收和更多的电子-空穴对, 使得该器件的 光响应度高达 $0.1 \mathrm{~A} / \mathrm{W}$, 量子产率为 $30 \%$ (图1(b)). 理 论计算显示, 单层 $\mathrm{MoS}_{2}$ 室温下的电子迁移率为 400 $\mathrm{cm}^{2} \mathrm{~V}^{-1} \mathrm{~s}^{-1[45]}$, 然而实验所获得的迁移率均低于 100 $\mathrm{cm}^{2} \mathrm{~V}^{-1} \mathrm{~s}^{-1[6,20 \sim 22]}$. 金属电极与 $\mathrm{MX}_{2}$ 之间的高接触电 阻是导致迁移率低于理论值的主要原因 ${ }^{[46]}$. 为此,
美国哥伦比亚大学Hone课题组 ${ }^{[47]}$ 以机械剥离单层石 墨烯作为机械剥离不同层厚 $\mathrm{MoS}_{2}$ 的接触电极, 构筑 了 graphene/ $\mathrm{MoS}_{2}$ 场效应晶体管, 并测得低温下 (12 $\mathrm{K})$ 六层 $\mathrm{MoS}_{2}$ 的霍尔迁移率高达 $34000 \mathrm{~cm}^{2} \mathrm{~V}^{-1} \mathrm{~s}^{-1}$, $\mathrm{MoS}_{2}$ 与石墨烯的接触电阻仅为 $1 \mathrm{k} \Omega \mu \mathrm{m}$ (图1(c)).

实现 $\mathrm{MX}_{2} /$ graphene层间异质结构在电子、光电子 和能源领域中的应用，可控制备大畴区、大面积、高 质量 $\mathrm{MX}_{2}$ /graphene层间异质结构是前提. 目前这类 异质结构的制备方法主要有3种：(1) 机械剥离 $\mathrm{MX}_{2}$

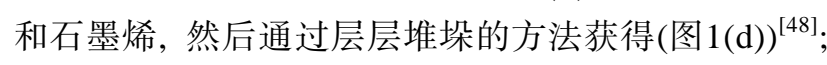
(2) 在CVD生长的石墨烯上, 通过 $\left(\mathrm{NH}_{4}\right)_{2} \mathrm{MX}_{4}$ 溶液的

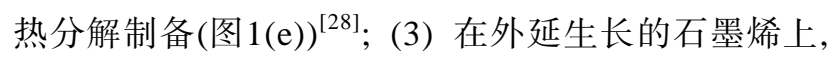
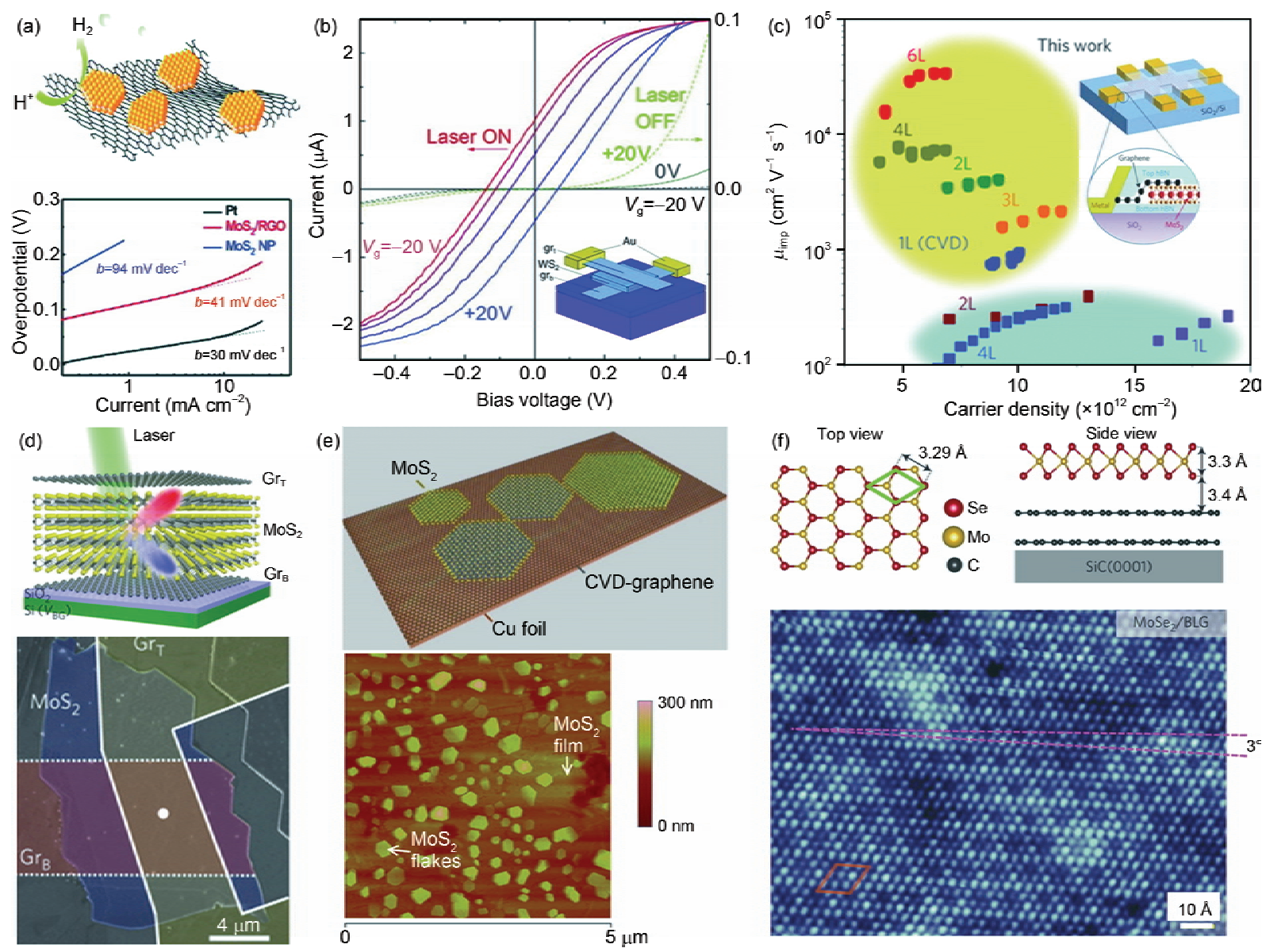

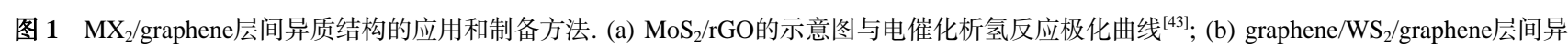

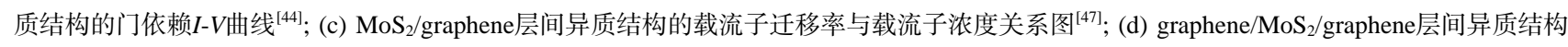
的示意图和扫描电子显微镜(scanning electron microscope, SEM) 图 ${ }^{[48]}$; (e) CVD graphene/铜簿上制备 $M_{0} S_{2}$ 的示意图和原子力显微镜(atomic force microscope, $\mathrm{AFM}$ ) 图 ${ }^{[28]}$; (f) 外延双层graphene/ $\mathrm{SiC}$ 上制备单层 $\mathrm{MoSe}_{2}$ 示意图和扫描隧道显微镜图(扫描条件: $V_{\mathrm{s}}=-0.9 \mathrm{~V}, I_{\mathrm{t}}=20 \mathrm{pA}, T=5 \mathrm{~K}$ ) ${ }^{[49]}$ Figure 1 Applications and syntheses methods of $\mathrm{MX}_{2} /$ graphene vertical heterostructures. (a) Schematic and polarization curves of $\mathrm{MoS} / \mathrm{rGO}$ in hydrogen evolution reaction ${ }^{[43]}$; (b) gate-dependent $I-V$ curves of graphene/ $\mathrm{WS}_{2} /$ graphene heterostructure ${ }^{[44]}$; (c) the relationship of mobility and carrier density of $\mathrm{MoS}_{2} /$ graphene heterostructure ${ }^{[47]}$; (d) schematic and SEM image of graphene/ $\mathrm{MoS}_{2} /$ graphene vertical heterostructure ${ }^{[48]}$; (e) schematic and AFM image of $\mathrm{MoS}_{2} / \mathrm{graphene} \mathrm{Cu}^{[28]}$; (f) schematic and STM image of monolayer $\mathrm{MoSe}_{2}$ on bilayer graphene $\left(V_{\mathrm{s}}=-0.9 \mathrm{~V}, I_{\mathrm{t}}=20 \mathrm{pA}, T=5 \mathrm{~K}\right)^{[49]}$ 
通过分子束外延的方法获得 $\mathrm{MX}_{2}$ (图 1(f) $)^{[49]}$. 然而, 以上制备方法均不同程度地存在缺陷. 所获得 $\mathrm{MX}_{2}$ 的畴区尺寸小、层厚不可控、界面易污染、制备工艺 复杂无法实现批量生产等. 因此, 寻找一种畴区尺寸/ 层厚可控、大面积、批量制备的生长工艺是目前材料 科学领域关注的焦点.

总之, $\mathrm{MX}_{2} /$ graphene层间异质结构, 利用了 $\mathrm{MX}_{2}$ 和石墨烯迥异的物理和化学性质, 在电子、光电子器 件、可再生能源储存和转化等领域具有潜在应用价 值. 可控制备、大畴区、大面积高质量 $\mathrm{MX}_{2} /$ graphene 层间异质结构是实现其实际应用的前提.

\section{$2 \mathrm{Au}$ 䈃上 $\mathrm{MoS}_{2} /$ graphene 层间异质结构 CVD制备及其在电催化析氢反应中的 应用}

$\mathrm{MX}_{2} /$ graphene层间异质结构的可控制备是实现 新奇物理性质研究和实际应用探索的首要条件. CVD是一种兼顾材料质量、制备效率和制备成本的 方案, 因而被认为是一种能够实现大畴区、大面积、 高质量二维材料批量生产和规模应用的最可能途径. 二维材料的 CVD生长过程中, 前驱物需要经由气相 引至生长基底表面, 使得生长体系的压力以及载气 的成分和流量均会对生长结果产生重要影响. 尽管 丰富的实验参数给体系优化带来了一定的复杂性, 却也提供了更大的调节空间, 使得在合适的参数组 合下能够获得令人意外的生长结果. 以下将详细阐 述 $\mathrm{Au}$ 䈃上 $\mathrm{MoS}_{2} / \mathrm{graphene}$ 层间异质结构的两步 CVD可 控制备、精密表征及该异质结构在电催化析氢反应中 的应用.

\section{$2.1 \mathrm{Au}$ 䇴上两步 CVD 制备 $\mathrm{MoS}_{2} /$ graphene 层间 异质结构}

由于 $\mathrm{MX}_{2}$ 生长过程中通常采用 $\mathrm{S}$ 粉作为前驱体, 而大多数金属箔材 (如铜、镍、铁) 在高温下均会和 $S$ 蒸气发生化学反应, 生成粉末状的金属硫化物. 因此 这些金属䈃材不适合作为 $\mathrm{MX}_{2}$ 的生长基底. $\mathrm{Au}$ 簿在 $\mathrm{S}$ 蒸气氛围下显示了非常强的化学惰性, 不会和 $\mathrm{S}$ 发生 化学反应, 仍然保持原有的形状和性质不被破坏, 因 而是一种非常优异的生长基底. 值得一提的是, 最近 武汉大学付磊课题组 ${ }^{[50]}$ 在较廉价的 $\mathrm{Ni}-\mathrm{Ga}$ 合金基底 上实现了 $\mathrm{MoS}_{2} / h-\mathrm{BN}$ 层间异质结构的可控制备, 进而
为 $\mathrm{MX}_{2}$ 的生长提供了更广泛的选择空间. 通过两步 $\mathrm{CVD}$ 方法在 $\mathrm{Au}$ 簿上实现了 $\mathrm{MoS}_{2} /$ graphene层间异质结 构的可控制备(图2(a) $)^{[32]}$. Au簿上石墨烯的常压CVD 生长过程: $\mathrm{Au}$ 篞被放置于内径为 1 英寸 $(1$ 英寸 $=2.54$ $\mathrm{cm}$ )的三温区管式炉(lindberg/blue M)中, 生长温度为 $900^{\circ} \mathrm{C}$, 生长时间为 $30 \mathrm{~min}$, 所用碳源为甲烷 (流量为 $5 \mathrm{sccm}\left(1 \mathrm{sccm}\right.$ 表示 $\left.\left.1 \mathrm{~mL} \mathrm{~min}^{-1}\right)\right)$, 载气为氞气(100 $\mathrm{sccm})$ 和氢气 $(10 \mathrm{sccm})$. 扫描电子显微镜表征显示, $\mathrm{Au}$ 䇴上获得了层厚均匀的石墨烯薄膜(深色区域为石 墨烯, 浅色区域为裸漏 $\mathrm{Au}$ 基底)(图2(b)). 拉曼光谱显 示, $\mathrm{Au}$ 上石墨烯的 $\mathrm{G}$ 峰和 $2 \mathrm{D}$ 峰分别位于 1589 和 2685 $\mathrm{cm}^{-1}, \mathrm{G}$ 峰和 $2 \mathrm{D}$ 峰强度比为 0.45 , 这表明所获得石墨烯 薄膜是单层的(图2(d)). 随后将 graphene/Au转移至 $\mathrm{MoS}_{2}$ 的生长管式炉中，利用低压 CVD的方法进行 $\mathrm{MoS}_{2}$ 的生长. Graphene/ $\mathrm{Au}$ 上 $\mathrm{MoS}_{2}$ 生长过程: 前驱体 $\mathrm{MoO}_{3}$ 和 graphene/Au分别被放置于内径为 1 英寸的三 温区管式炉中，在 $\mathrm{MoO}_{3}$ 和 Graphene/Au中间施加隔 热套, 对 $\mathrm{MoO}_{3}$ 和 graphene/Au的温度分别进行控制 (放置 $\mathrm{MoO}_{3}$ 温区的温度固定为 $530^{\circ} \mathrm{C}$, graphene/ $\mathrm{Au}$ 的 温度为 $680^{\circ} \mathrm{C}$ ); 升华 $\mathrm{S}$ 粉放置于管式炉的外部, 并用 加热带进行加热 (加热温度固定为 $102^{\circ} \mathrm{C}$ ); 当 $\mathrm{MoO}_{3}$ 被 加热到 $530^{\circ} \mathrm{C}$ 时, 会被气化的 $\mathrm{S}$ 粉部分还原形成易挥 发的低值氧化物 $\mathrm{MoO}_{3-x}$, 随后 $\mathrm{MoO}_{3-x}$ 和进一步被硫 化形成的 $\mathrm{MoS}_{2}$ 被载气( $\mathrm{Ar}$ ) 输运到 graphene/ $\mathrm{Au}$ 上. $\mathrm{SEM}$ 表征显示, graphene/Au上形成了三角形的 $\mathrm{MoS}_{2}$, 畴区尺寸为 $200 \mathrm{~nm}$ (深色三角形区域为 $\mathrm{MoS}_{2}$, 浅色区 域为 graphene/Au)(图2(c)). 图2(d)中的拉曼光谱显示, $\mathrm{MoS}_{2}$ 的两个特征峰 $\left(E_{2 \mathrm{~g}}^{1}\right.$ 和 $\left.A_{1 \mathrm{~g}}\right)$ 分别位于 385.5 和 405.7 $\mathrm{cm}^{-1}$, 两个特征峰的位置间距为 $20.2 \mathrm{~cm}^{-1}$. 这两个特 征峰的间距与前期报道的绝缘基底上制备的单层

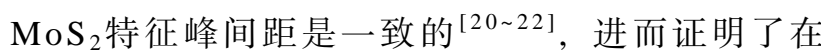
graphene/ $\mathrm{Au}$ 上获得了单层 $\mathrm{MoS}_{2}$. 需要指出的是, 通 过两步 $\mathrm{CVD}$ 方法在 $\mathrm{Au}$ 馢上制备的 $\mathrm{MoS}_{2} /$ graphene层间 异质结构可以完整无损地转移到其他任意衬底上(图 2(e)), 为该异质结构的进一步应用提供了前提. $\mathrm{Au}$ 䈱 上 $\mathrm{MoS}_{2} /$ graphene的转移方法: 对载有 $\mathrm{MoS}_{2} /$ graphene 的 $\mathrm{Au}$ 箔表面进行 $3000 \mathrm{r} / \mathrm{min}$ 的旋涂样品支撑膜(聚甲 基丙烯酸甲酯(poly(methylmethacrylate), PMMA)), 而后在 $170^{\circ} \mathrm{C}$ 的加热台上烘干; 将烘干后的样品置于 $\mathrm{Au}$ 䈃刻蚀剂中进行 $\mathrm{Au}$ 基底的刻蚀(金筞刻蚀剂为 $\mathrm{KI}+\mathrm{I}_{2}+\mathrm{H}_{2} \mathrm{O}$, 质量比为 $1: 4: 40$ ); 将 PMMA支撑的 $\mathrm{MoS}_{2} /$ graphene从 $\mathrm{Au}$ 刻蚀剂中捞出, 并在去离子水中 

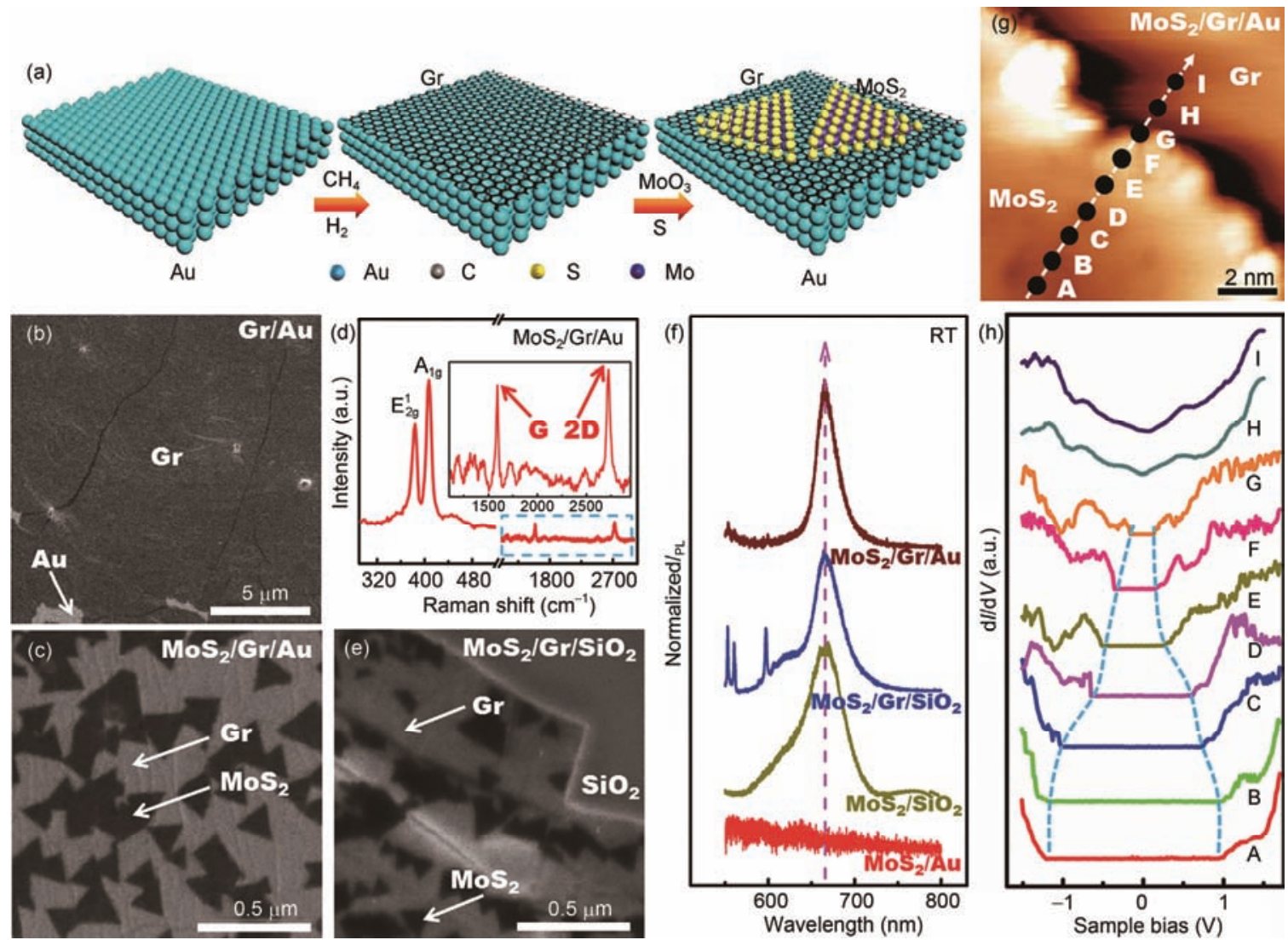

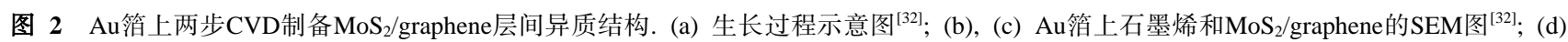

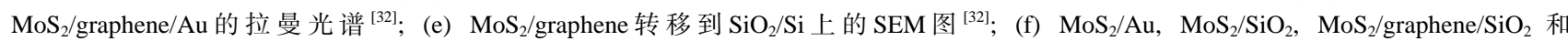
$\mathrm{MoS}_{2} / \mathrm{graphene} / \mathrm{Au}$ 的光致发光谱 ${ }^{[32]} ; \mathrm{MoS}_{2} / \mathrm{graphene/Au}$ 的扫描隧道显微镜(STM)图 $\left(\mathrm{g}\right.$ )和扫描隧道(STS)谱(h)(扫描条件: $V_{\mathrm{s}}=-1.2 \mathrm{~V}, I_{\mathrm{t}}=0.2 \mathrm{nA}$, $T=78 \mathrm{~K}, 10 \mathrm{mV}, 932 \mathrm{~Hz})^{[34]}$

Figure 2 Two-step CVD synthesis of $\mathrm{MoS}_{2} /$ graphene heterostructure on Au foils. (a) Schematic illustration of the CVD growth process ${ }^{[32]}$; (b), (c) SEM images of graphene and $\mathrm{MoS}_{2} /$ graphene vertical heterostructure on Au foils ${ }^{[32]}$; (d) Raman spectrum of $\mathrm{MoS}_{2} / \mathrm{graphene} / \mathrm{Au}^{[32]}$; (e) SEM image of $\mathrm{MoS}_{2} /$ graphene vertical heterostructure transferred onto $\mathrm{SiO}_{2} / \mathrm{Si}$ substrate ${ }^{[32]}$; (f) $\mathrm{PL}$ spectra of $\mathrm{MoS}_{2} / \mathrm{Au}, \mathrm{MoS}_{2} / \mathrm{SiO}_{2}, \mathrm{MoS}_{2} / \mathrm{graphene} / \mathrm{SiO}_{2}$, and $\mathrm{MoS}_{2} /$ graphene/ $/ \mathrm{Au}^{[32]}$; STM image and $(\mathrm{g}) \mathrm{STS}$ spectra of (h) monolayer $\mathrm{MoS}_{2}$ on graphene $\left(V_{\mathrm{s}}=-1.2 \mathrm{~V}, I_{\mathrm{t}}=0.2 \mathrm{nA}, T=78 \mathrm{~K}, 10 \mathrm{mV}, 932 \mathrm{~Hz}\right)^{[34]}$

洗涤除去残留的 $\mathrm{KI}$ 和 $\mathrm{I}_{2}$; 利用 $\mathrm{SiO}_{2} / \mathrm{S}$ 将洗涤干净的样 品捞出, 并在红外灯下烘烤; 最后利用丙酮蒸气除 去PMMA支撑膜. 利用这种方法成功地将 $\mathrm{Au}$ 䇴上生 长的 $\mathrm{MoS}_{2} /$ graphene转移到 $\mathrm{SiO}_{2} / \mathrm{Si}$ 基底上, 如图 2(e) 所示.

利用PL光谱表征了所制备 $\mathrm{MoS}_{2} /$ graphene的晶体 质量(图 2(f)). 有趣的是, 当 $\mathrm{MoS}_{2}$ 生长在 $\mathrm{Au}$ 簿上时, 其PL信号被完全淬灭. 当 $\mathrm{MoS}_{2}$ 生长在 graphene/Au管 上时, 其PL信号则被明显地观察到. 这说明, 石墨烯 的插层显著削弱了 $\mathrm{MoS}_{2}$ 与 $\mathrm{Au}$ 䇴之间的界面相互作 用, 进而实现了本征 $\mathrm{MoS}_{2}$ PL信号的直接观测. 此外, $\mathrm{MoS}_{2} /$ graphene/Au的PL特征峰半高峰宽仅为 $40 \mathrm{meV}$, 远低于 $\mathrm{MoS}_{2} / \mathrm{SiO}_{2}(63 \mathrm{meV})$, 表明利用两步 CVD方法 制备的 $\mathrm{MoS}_{2} /$ graphene层间异质结构, 界面干净/本征,
没有任何有机物/无机物的污染和光散色现象发生. 与此同时, 利用低温 STM/STS研究了 $\mathrm{MoS}_{2} /$ graphene 的原子尺度形貌和局域电子结构. 图 2(g) 显示了 graphene/ $/ \mathrm{Au}$ 上 $\mathrm{MoS}_{2}$ 畴区边界处的形貌. 理论计算表 明, $\mathrm{MX}_{2}$ 的畴区边界处, 费米面附近电子态密度增加, 在 STM图像中表现为高亮衬度 ${ }^{[51]}$. STS 表征显示, $\mathrm{MoS}_{2}$ 的畴区内部, 带隙宽度为 $2.2 \mathrm{eV}$, 导带和价带分 别位于 +1.2 和 $-1.0 \mathrm{eV}$. 这种导带和价带近乎对称的 现象说明, 石墨烯的插层明显减弱了 $\mathrm{Au}$ 䈃对单层 $\mathrm{MoS}_{2}$ 的电子掺杂, 进而获得了本征 $\mathrm{MoS}_{2}$ 的能带结构. 有趣的是, $\mathrm{MoS}_{2}$ 的畴区边界处, 带隙宽度明显减小, 最终达到 $0.3 \mathrm{eV}$, 这种现象表明, $\mathrm{MoS}_{2}$ 的畴区边界具 有金属特性. $\mathrm{MoS}_{2}$ 畴区边界处带隙宽度的减小, 有望 实现更加高效的电催化析氢反应. 


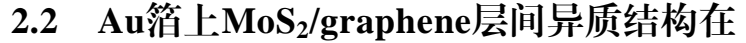 电催化析氢反应中的应用}

依据前期理论计算结果, 氢离子在 $\mathrm{MoS}_{2} / \mathrm{gra}-$ phene活性位点的吸附势垒明显低于单一 $\mathrm{MoS}_{2}{ }^{[42]}$, 因 此, $\mathrm{MoS}_{2}$ /graphene有望实现更加高效的电催化产氢. 此外, $\mathrm{MoS}_{2}$ 电催化析氢反应活性位点位于金属终止 的边缘处, 表面没有电催化活性 ${ }^{[52]}$. 根据前期 $\mathrm{MoS}_{2} /$ graphene的扫描透射电子显微镜表征结果, $\mathrm{MoS}_{2}$ 的畴 区边界是 $\mathrm{Mo}$ 终止的边缘类型 ${ }^{[32]}$. 基于这一考量, 通 过调控 graphene/ $\mathrm{Au}$ 与 $\mathrm{MoO}_{3}$ 之间的距离获得了覆盖 度从 $10 \%$ 到 $80 \%$ 的样品. 增大单位面积 $\mathrm{MoS}_{2}$ 的覆盖 度可以进一步增加活性位点的密度, 进而提高其电 催化析氢性能.

图3(a)显示了 $\mathrm{MoS}_{2} / \mathrm{graphene} / \mathrm{Au}$ 和 $\mathrm{MoS}_{2} / \mathrm{Au}$ 电催 化析氢反应的示意图, 催化活性位点均位于 $\mathrm{MoS}_{2}$ 的 边缘处. 图3(b), (c) 分别显示了具有相同覆盖度 ( $65 \%)$ 的 $\mathrm{MoS}_{2} / \mathrm{graphene} / \mathrm{Au}$ 和 $\mathrm{MoS}_{2} / \mathrm{Au}$ 的形貌. 将这 两种样品直接进行电催化析氢反应, 并对比其催化 性能. 极化曲线显示, $\mathrm{MoS}_{2} / \mathrm{graphene} / \mathrm{Au}$ 和 $\mathrm{MoS}_{2} / \mathrm{Au}$
的开启电压分别为 90 和 $110 \mathrm{mV}$ (图3(d)). 这说明 $\mathrm{MoS}_{2} / \mathrm{graphene} / \mathrm{Au}$ 相比 $\mathrm{MoS}_{2} / \mathrm{Au}$ ，在更低的过电位下 实现了电催化析氢. 塔菲尔斜率是反映催化剂催化 效果的重要参数, 受电催化析氢反应中决速步的影 响 ${ }^{[43]}$. 在酸性电解液中, 电催化析氢反应要经历 3 个 过程: 放电过程(1)、电化学脱附过程(2)、再复合过 程(3):

$$
\begin{gathered}
\mathrm{H}_{3} \mathrm{O}^{+}+\mathrm{e}^{-} \rightarrow \mathrm{H}_{\mathrm{ads}}+\mathrm{H}_{2} \mathrm{O}, \\
\mathrm{H}_{\mathrm{ads}}+\mathrm{H}_{3} \mathrm{O}^{+}+\mathrm{e}^{-} \rightarrow \mathrm{H}_{2}+\mathrm{H}_{2} \mathrm{O}, \\
\mathrm{H}_{\mathrm{ads}}+\mathrm{H}_{\mathrm{ads}} \rightarrow \mathrm{H}_{2} .
\end{gathered}
$$

当塔菲尔斜率为 $120 \mathrm{mV} \mathrm{dec}{ }^{-1}$ 时, 决速步为放电 过程(1); 塔菲尔斜率为 $30 \mathrm{mV} \mathrm{dec}$ 时, 决速步为再 复合过程(3); 塔菲尔斜率为 $40 \mathrm{mV} \mathrm{dec}{ }^{-1}$ 时, 决速步 为电化学脱附过程 (2). 图3(e)中塔菲尔曲线显示,

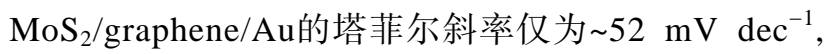
远小于 $\mathrm{MoS}_{2} / \mathrm{Au}\left(\sim 70 \mathrm{mV} \mathrm{dec}{ }^{-1}\right)$. 表明 $\mathrm{MoS}_{2} /$ graphene/ $\mathrm{Au}$ 相比 $\mathrm{MoS}_{2} / \mathrm{Au}$, 具有更快的电催化析氢反应速度. 交换电流密度是反映电催化析氢效率的另一个重要 参数. 为进一步对比 $\mathrm{MoS}_{2} / \mathrm{graphene} / \mathrm{Au}$ 和 $\mathrm{MoS}_{2} / \mathrm{Au}$ 的

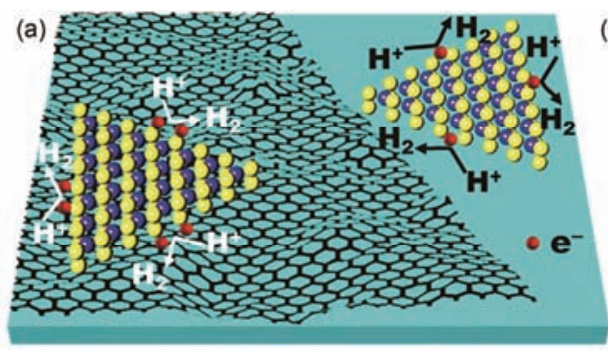

(b)
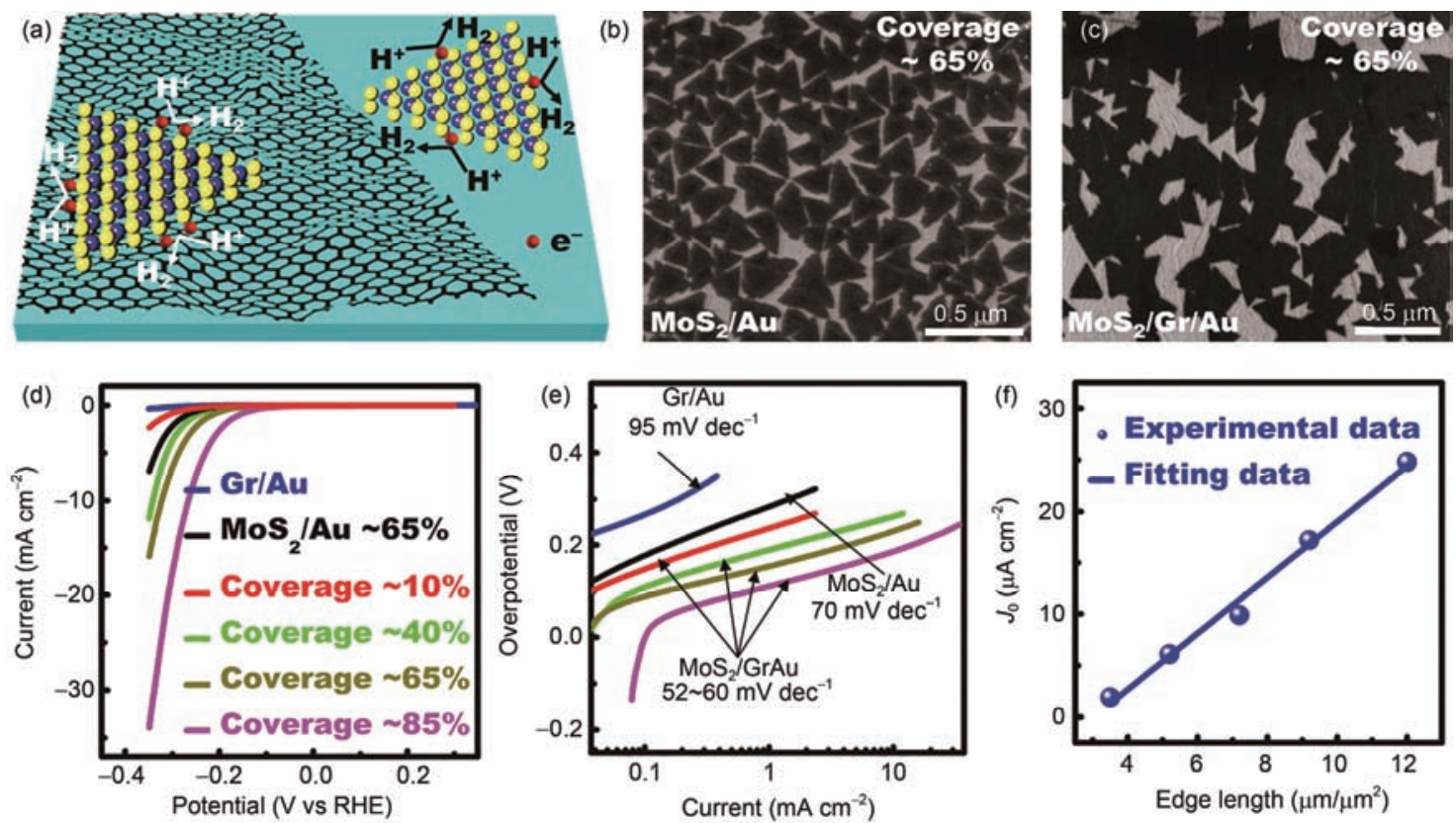

图 $3 \mathrm{Au}$ 䈃上 $\mathrm{MoS}_{2} / \mathrm{graphene}$ 层间异质结构在电催化析氢反应中的应用. (a) $\mathrm{MoS}_{2} / \mathrm{graphene} / \mathrm{Au}$ 和 $\mathrm{MoS}_{2} / \mathrm{Au}$ 的电催化析氢反应示意图; (b), (c) $\mathrm{MoS}_{2} / \mathrm{graphene} / \mathrm{Au}$ 和 $\mathrm{MoS}_{2} / \mathrm{Au}$ 的扫描电子显微镜图; $\mathrm{MoS}_{2} / \mathrm{graphene} / \mathrm{Au}, \mathrm{MoS}_{2} / \mathrm{Au}$ 和graphene/Au的极化曲线(d)和塔菲尔曲线(e); (f) 交换电流密 度与 $\mathrm{MoS}_{2}$ 边缘长度的关系图 ${ }^{[34]}$

Figure 3 The application of $\mathrm{MoS}_{2} /$ graphene/Au in hydrogen evolution reaction. (a) Schematic illustrations of hydrogen evolution reaction of $\mathrm{MoS}_{2} /$ graphene/Au and $\mathrm{MoS}_{2} / \mathrm{Au}$; (b), (c) SEM images of $\mathrm{MoS}_{2} /$ graphene/Au and $\mathrm{MoS}_{2} / \mathrm{Au}$; polarization curves (d) and corresponding Tafel plots (e) of $\mathrm{MoS}_{2} / \mathrm{Gr} / \mathrm{Au}, \mathrm{MoS}_{2} / \mathrm{Au}$ and $\mathrm{Gr} / \mathrm{Au}$, respectively; (f) statistical distributions of the exchange current density as a function of the edge length of MoS 2 on $\mathrm{Gr} / \mathrm{Au}^{[34]}$ 
析氢效果, 根据塔菲尔曲线, 计算了具有相同覆盖度 ( $65 \%)$ 的 $\mathrm{MoS}_{2} /$ graphene/ $\mathrm{Au}$ 和 $\mathrm{MoS}_{2} / \mathrm{Au}$ 的交换电流密 度. 结果显示, $\mathrm{MoS}_{2} / \mathrm{graphene} / \mathrm{Au}$ 的交换电流密度为 $24.8 \mu \mathrm{A} \mathrm{cm}^{-2}$, 远高于 $\mathrm{MoS}_{2} / \mathrm{Au}\left(15.7 \mu \mathrm{A} \mathrm{cm}^{-2}\right)$, 进一 步表明 $\mathrm{MoS}_{2} / \mathrm{graphene} / \mathrm{Au}$ 具有更高的电催化析氢效 果. 此外, 通过统计 $\mathrm{MoS}_{2} /$ graphene/Au的交换电流密 度与单位面积 $\mathrm{MoS}_{2}$ 边缘长度的关系, 结果显示两者 之间具有线性依赖性(图3(f)), 这说明 $\mathrm{MoS}_{2} /$ graphene/ $\mathrm{Au}$ 的电催化析氢反应活性位点位于 $\mathrm{MoS}_{2}$ 边缘处.

总之, 本课题组利用两步 CVD方法实现了 $\mathrm{Au}$ 箔 上 $\mathrm{MoS}_{2} /$ graphene层间异质结构的可控制备, 并获得 了本征异质界面. 利用低温STM/STS获得了 $\mathrm{MoS}_{2}$ 原 子尺度形貌和局域电子结构, 为理解 CVD制备的 $\mathrm{MoS}_{2} /$ graphene层间异质结构的晶体质量、能带结构 和器件性能提供了直接的实验依据. 将 $\mathrm{Au}$ 簿上制备 的 $\mathrm{MoS}_{2} /$ graphene层间异质结构直接应用于电催化析 氢反应, 获得了高效的电催化析氢效果. 需要指出的 是, 进一步发展廉价金属衬底上高质量 $\mathrm{MX}_{2} / \mathrm{gra}-$ phene层间异质结构的CVD可控制备是实现其规模化 应用的前提. 金属合金有望实现抗硫化作用, 进而为 $\mathrm{MX}_{2}$ 的 CVD生长提供更加广阔的选择自由度.

\section{$3 \mathbf{M X}_{2} / \mathbf{M X}_{2}$ 层间异质结构的新奇物理 性质和制备方法}

理论计算表明, $\mathrm{MX}_{2} / \mathrm{MX}_{2}$ 层间异质结构是一种 II 型半导体, 导带和价带分别位于两种 $\mathrm{MX}_{2}$ 材料上, 电子-空穴对能够实现自发分离 ${ }^{[15]}$. 这种异质结构在 强界面耦合 (strong interlayer coupling) ${ }^{[53]}$ 、超快电荷 传输(ultrafast charge transfer) ${ }^{[13,54]}$ 、界面等离子共振 (plasma oscillation) $^{[13]}$ 、层间激子自旋谷极化(interlayer exciton spin-valley polarization) ${ }^{[14]}$ 和光伏器件(photovoltaic device $)^{[55 \sim 58]}$ 等领域具有广泛的研究价值. 制备 高质量本征 $\mathrm{MX}_{2} / \mathrm{MX}_{2}$ 层间异质结构是进行这些新奇 物理性质研究的首要条件.

美国加州大学伯克利分校Wang课题组 ${ }^{[59]}$ 以转移 $\mathrm{CVD}$ 生长的单层 $\mathrm{MoS}_{2}$ 和单层 $\mathrm{WS}_{2}$ 构筑了 $\mathrm{MoS}_{2} / \mathrm{WS}_{2}$ 层 间异质结构, 如图4(a)所示. 他们利用瞬态吸收光谱 观察到 $\mathrm{MoS}_{2} / \mathrm{WS}_{2}$ 异质界面处的超快电荷传输现象, 光激化空穴由 $\mathrm{MoS}_{2}$ 层传输至 $\mathrm{WS}_{2}$ 层仅需 $50 \mathrm{fs}$. 美国 华盛顿大学Xu课题组 ${ }^{[60]}$ 利用机械剥离单层 $\mathrm{MoSe}_{2}$ 和 单层 $\mathrm{WSe}_{2}$ 构筑了 $\mathrm{MoSe}_{2} / \mathrm{WSe}_{2}$ 层间异质结构(图4(b)). 通过时间分辨PL光谱观察到了层间激子跃迁能 $(\sim 1.35 \mathrm{eV})$, 这种层间激子的PL寿命为 $1.8 \mathrm{~ns}$, 比层内 激子 PL寿命高 1 个数量级. 具有长寿命层间激子的 $\mathrm{MoSe}_{2} / \mathrm{WSe}_{2}$ 层间异质结构在二维激光、光伏器件和 发光二极管等领域具有潜在应用价值. 此外, Xu课题 组 ${ }^{[14]}$ 利用圆偏振光泵浦的方法, 在 $\mathrm{WSe}_{2} / \mathrm{MoSe}_{2}$ 层间 异质结构中实现了层间激子自旋谷极化, 并测得谷 寿命为 $40 \mathrm{~ns}$. 与此同时, 美国宾夕法尼亚州立大学 Robinson课题组 ${ }^{[61]}$ 以 $\mathrm{SiC}$ 外延三层石墨烯为生长衬 底, 利用两步金属有机化学气相沉积的方法实现了 $\mathrm{MoS}_{2} / \mathrm{WSe}_{2}$ 层间异质结构的可控制备. 随后, 他们利 用导电 $\mathrm{AFM}$ 研究了该异质结构的电学特性. 令人惊 奇的是, $\mathrm{MoS}_{2} / \mathrm{WSe}_{2}$ 层间异质结构显示出了新奇的负 微分电阻(negative differential resistance, NDR)效应, 表明该异质结构具有超高的晶体质量和本征的异质 界面.

实现 $\mathrm{MX}_{2} / \mathrm{MX}_{2}$ 层间异质结构新奇物理特性的直 接观测, 获得高质量和本征 $\mathrm{MX}_{2} / \mathrm{MX}_{2}$ 层间异质结构 是先决条件. 目前主流的制备方法有以下3类: (1) 机 械剥离不同带隙宽度的单层 $\mathrm{MX}_{2}$ 材料, 然后通过层 层堆垛的方法获得(图4(d) $)^{[53] ;}$; (2) 层层转移和堆垛

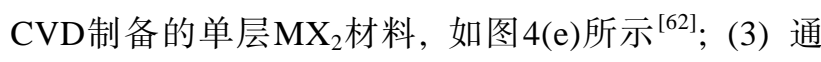
过一步 CVD的方法直接生长获得(图4(f) $)^{[30]}$. 不可否 认, 机械剥离和转移法操作简单、制备的样品质量 高、新鲜解理出的表面较为洁净, 适用于各种基础结 构表征、光谱研究、电子和光电原型器件的构建. 然 而, 这种方法具有很多局限性, 如制备效率低不具有 可扩展性、晶体层厚、均匀性不佳、畴区尺寸受限、 基底表面和异质界面的高分子残留污染等。一步 CVD的方法, 由于异质结构制备过程中, 所有的前 驱物在生长气氛中共存, 所获得 $\mathrm{MX}_{2} / \mathrm{MX}_{2}$ 极易形成 合金相或界面处发生元素互混, 无法获得本征的异 质结构. 因此, 寻找一种可控地制备本征 $\mathrm{MX}_{2} / \mathrm{MX}_{2}$ 层间异质结构的生长工艺是材料制备领域亟需解决 的科学问题.

\section{4 两步法 CVD选择性制备 $\mathrm{MoS}_{2} / \mathrm{WS}_{2}$ 和 $\mathrm{WS}_{2} / \mathrm{MoS}_{2}$ 层间异质结构与光催化析氢 反应特性}

$\mathrm{MoS}_{2} / \mathrm{WS}_{2}$ 和 $\mathrm{WS}_{2} / \mathrm{MoS}_{2}$ 层间异质结构是 II 型半 导体, 在光电器件和光催化析氢等领域具有潜在的 应用价值. 大畴区、大面积可控制备本征 $\mathrm{MoS}_{2} / \mathrm{WS}_{2}$ 和 $\mathrm{WS}_{2} / \mathrm{MoS}_{2}$ 层间异质结构是先决条件. 本小节将详 

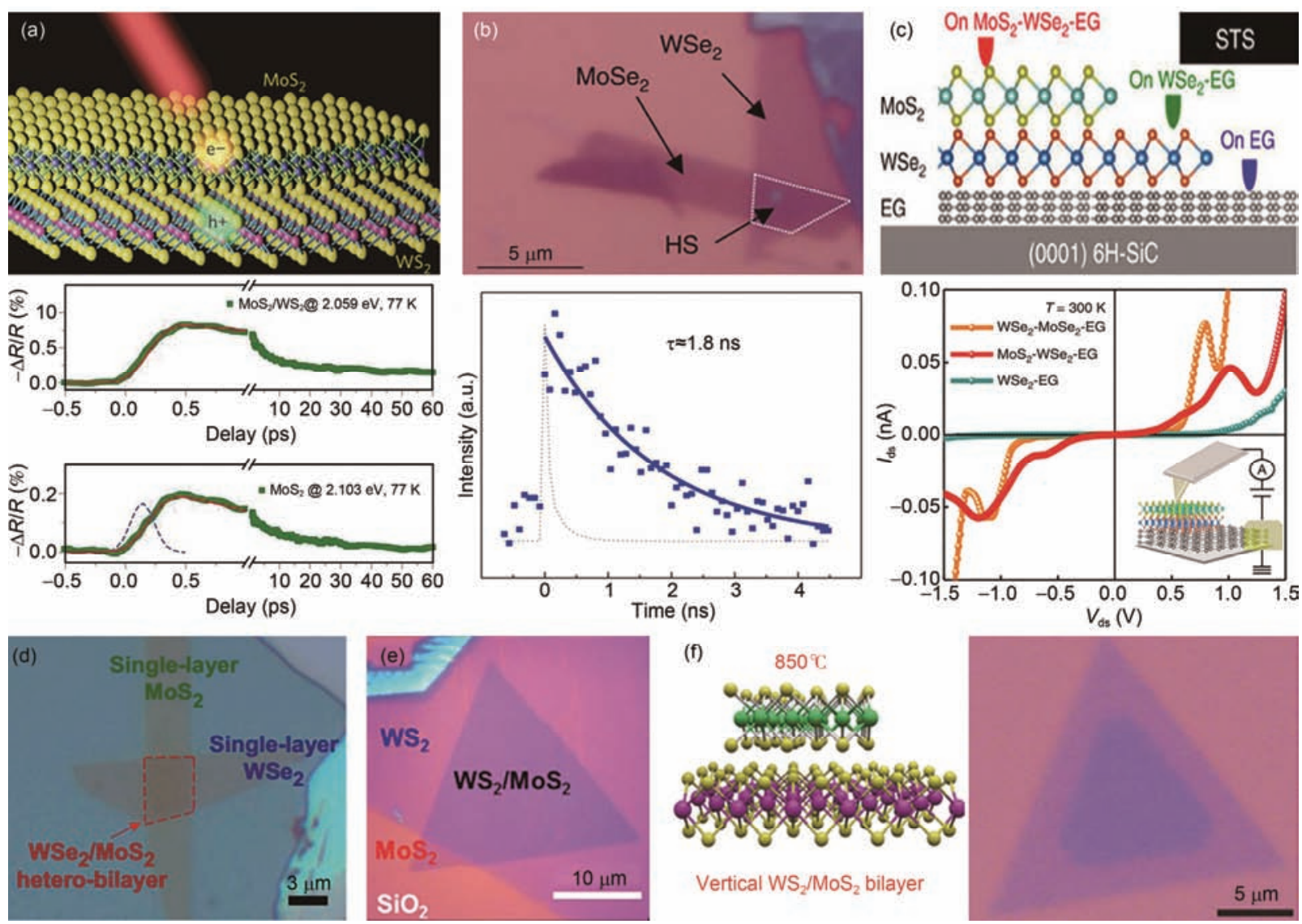

图 $4 \mathrm{MX}_{2} / \mathrm{MX}_{2}$ 层间异质结构的新奇物理性质和制备方法. (a) $\mathrm{MoS}_{2} / \mathrm{WS}_{2}$ 层间异质结构示意图和瞬态吸收光谱 ${ }^{[59]} ;$ (b) $\mathrm{MoSe}_{2} / \mathrm{WSe}_{2}$ 层间异质结 构的光学图和时间分辨光致发光光谱 ${ }^{[60] ; ~(c) ~} \mathrm{SiC}$ 外延石墨烯上制备的 $\mathrm{MoS}_{2} / \mathrm{WSe}_{2}$ 层间异质结构示意图和 $I-V$ 图 ${ }^{[61]} ;(\mathrm{d}),(\mathrm{e}) \mathrm{WSe}_{2} / \mathrm{MoS}_{2}{ }^{[53]}$ 和 $\mathrm{WS}_{2} / \mathrm{MoS}_{2}{ }^{[62]}$ 层间异质结构的光学图; (f) $\mathrm{WS}_{2} / \mathrm{MoS}_{2}$ 层间异质结构的示意图和光学图 ${ }^{[30]}$

Figure 4 Physical properties and syntheses methods of $\mathrm{MX}_{2} / \mathrm{MX}_{2}$ vertical heterostructures. (a) Illustration and transient absorption spectra of $\mathrm{MoS}_{2} / \mathrm{WS}_{2}$ vertical heterostructure ${ }^{[59]}$; (b) optical image and time-resolved PL spectrum of $\mathrm{MoSe}_{2} / \mathrm{WSe}_{2}$ vertical heterostructure ${ }^{[60]}$; (c) illustration and experimental $I$ - $V$ traces of $\mathrm{MoS}_{2} / \mathrm{WSe}_{2}$ vertical heterostructure ${ }^{[61]}$; (d), (e) optical images of $\mathrm{WSe}_{2} / \mathrm{MoS}_{2}{ }^{[53]}$ and $\mathrm{MoS}_{2} / \mathrm{WS}_{2}{ }^{[62]}$ vertical heterostructures; (f) illustration and optical image of $\mathrm{WS}_{2} / \mathrm{MoS}_{2}$ vertical heterostructure ${ }^{[30]}$

细论述利用两步CVD方法选择性地制备 $\mathrm{MoS}_{2} / \mathrm{WS}_{2}$ 和 $\mathrm{WS}_{2} / \mathrm{MoS}_{2}$ 层间异质结构, 并展示了这两类异质结构 在光催化析氢反应中的应用.

\section{1 两步CVD法制备 $\mathrm{MoS}_{2} / \mathrm{WS}_{2}$ 和 $\mathrm{WS}_{2} / \mathrm{MoS}_{2}$ 层间 异质结构}

一步 CVD方法制备 $\mathrm{MX}_{2} / \mathrm{MX}_{2}$ 层间异质结构, 由 于前驱体在生长气氛中共存, 所获得异质结构多为 合金相或异质界面处发生元素互混, 无法制备本征 $\mathrm{MX}_{2} / \mathrm{MX}_{2}$ 层间异质结构. 沙特阿拉伯王国阿卜杜拉 国王科技大学 $\mathrm{Li}$ 课题组 ${ }^{[55]}$ 提出将不同 $\mathrm{MX}_{2}$ 材料的制 备过程分离在不同的生长体系中完成, 进而在蓝宝 石衬底上获得了本征 $\mathrm{WSe}_{2}-\mathrm{MoS}_{2}$ 面内异质结构. 随 后, 美国莱斯大学Ajayan课题组 ${ }^{[63]}$ 利用同样的制备方
法在 $\mathrm{SiO}_{2} / \mathrm{Si}$ 基底上制备了 $\mathrm{WSe}_{2} / \mathrm{MoSe}_{2}$ 层间异质结构. 本课题组 ${ }^{[35]}$ 利用两步 CVD方法实现了 $\mathrm{Au}$ 箔上 $\mathrm{MoS}_{2} / \mathrm{WS}_{2}$ 和 $\mathrm{WS}_{2} / \mathrm{MoS}_{2}$ 层间异质结构的选择性制备. $\mathrm{Au}$ 䇴上 $\mathrm{MoS}_{2} / \mathrm{WS}_{2}$ 层间异质结构的制备过程: 前驱体 $\mathrm{WO}_{3}$ 和生长衬底 $\mathrm{Au}$ 箔分别放置于内径为 1 英寸的三 温区管式炉中, $\mathrm{WO}_{3}$ 和 $\mathrm{Au}$ 䈃的温度均设定为 $880^{\circ} \mathrm{C}$, 升华 $\mathrm{S}$ 粉放置于管式炉的外部, 利用加热带加热至 $102^{\circ} \mathrm{C}$, 当 $\mathrm{WO}_{3}$ 被加热到 $880^{\circ} \mathrm{C}$ 时, 会被气化的 $\mathrm{S}$ 部分 还原形成易挥发的低值氧化物 $\mathrm{WO}_{3-x}$, 随后 $\mathrm{WO}_{3-x}$ 和 进一步被硫化形成的 $\mathrm{WS}_{2}$ 被载气( $\mathrm{Ar}$ )输运到 $\mathrm{Au}$ 馢上. 通过调控生长时间以及 $\mathrm{Au}$ 簿和 $\mathrm{WO}_{3}$ 之间的距离, 实 现了满单层 $\mathrm{WS}_{2}$ 薄膜的可控制备. 随后将满单层 $\mathrm{WS}_{2} / \mathrm{Au}$ 簿转移至另一个管式炉中进行单层 $\mathrm{MoS}_{2}$ 的生 长. 其生长过程与 $\mathrm{WS}_{2}$ 相似, 仅需将 $\mathrm{WO}_{3}$ 前驱体替换 
成 $\mathrm{MoO}_{3}$, 源和 $\mathrm{WS}_{2} / \mathrm{Au}$ 的温度分别设定为 530 和 $680^{\circ} \mathrm{C}$, 最终获得了 $\mathrm{MoS}_{2} / \mathrm{WS}_{2}$ 层间异质结构, 如图 $5(\mathrm{a})$ 所示. 同样地, 改变 $\mathrm{MoS}_{2}$ 和 $\mathrm{WS}_{2}$ 的生长顺序, 实 现了 $\mathrm{WS}_{2} / \mathrm{MoS}_{2}$ 层间异质结构的可控制备.

利用 SEM 对所获得 $\mathrm{MoS}_{2} / \mathrm{WS}_{2}$ 和 $\mathrm{WS}_{2} / \mathrm{MoS}_{2}$ 层间 异质结构的形貌进行表征, 结果表明, 上层的 $\mathrm{MoS}_{2}$ 和 $\mathrm{WS}_{2}$ 均为三角形, 畴区尺寸为 $200 \mathrm{~nm}$ (图5(b), (c)). 透射电子显微镜表征显示, $\mathrm{MoS}_{2} / \mathrm{WS}_{2}$ 和 $\mathrm{WS}_{2} / \mathrm{MoS}_{2}$ 层 间异质结构的衍射斑点均为一套, 表明这两类层间 异质结构均是通过外延生长模式获得的 ${ }^{[55]}$. 需要指 出的是, 扫描透射电子显微镜显示, $\mathrm{WS}_{2}$ 生长在 $\mathrm{MoS}_{2}$ 上时, 两种 $\mathrm{MX}_{2}$ 材料以 $\mathrm{A}-\mathrm{B}$ 形式堆垛 (W 原子堆垛在 $\mathrm{MoS}_{2}$ 层的 $\mathrm{S}$ 原子上, $\mathrm{WS}_{2}$ 层的 $\mathrm{S}$ 原子堆垛在 $\mathrm{Mo}$ 原子 上); $\mathrm{MoS}_{2}$ 生长在 $\mathrm{WS}_{2}$ 上时, 两种 $\mathrm{MX}_{2}$ 材料则是 $\mathrm{A}-\mathrm{A}$ 堆 垛形式( $\mathrm{Mo}$ 原子堆垛在 $\mathrm{W}$ 原子上, $\mathrm{MoS}_{2}$ 层的 $\mathrm{S}$ 原子堆 垛在 $\mathrm{WS}_{2}$ 的 $\mathrm{S}$ 原子上), 如图 5(d), (e)所示. 此外, 由于 $\mathrm{MoS}_{2}$ 和 $\mathrm{WS}_{2}$ 分别在两个体系中制备, 所获得 $\mathrm{MoS}_{2}$ / $\mathrm{WS}_{2}$ 和 $\mathrm{WS}_{2} / \mathrm{MoS}_{2}$ 均是本征的异质结构, 没有合金相 和元素互混现象发生. 这为进一步研究本征 $\mathrm{MoS}_{2}$ / $\mathrm{WS}_{2}$ 和 $\mathrm{WS}_{2} / \mathrm{MoS}_{2}$ 层间异质结构的新奇物理化学性质
提供了材料基础. 与此同时, 中国科学院物理研究所 张广宇课题组 ${ }^{[64]}$ 利用两步 $\mathrm{CVD}$ 方法在 $\mathrm{SiO}_{2} / \mathrm{Si}$ 衬底上 实现了 $\mathrm{MoS}_{2} / \mathrm{WS}_{2}$ 层间异质结构的可控制备(图 5(f), (g)). 他们利用拉曼光谱研究发现, 这种层间异质机 构具有强界面耦合和超快电荷传输特性.

综上可知, 利用两步 CVD方法可以在任意基底 上实现本征 $\mathrm{MX}_{2} / \mathrm{MX}_{2}$ 层间异质结构的可控制备, 为 更深人地研究这种异质结构的新奇物理性质提供了 前提.

\section{2 $\mathrm{MoS}_{2} / \mathrm{WS}_{2}$ 和 $\mathrm{WS}_{2} / \mathrm{MoS}_{2}$ 层间异质结构的层间 激子跃迁能和局域电学性质}

理论计算表明, $\mathrm{MX}_{2} / \mathrm{MX}_{2}$ 层间异质结构是一种 II 型半导体，导带和价带分别位于不同的 $\mathrm{MX}_{2}$ 材料 上 $^{[15]}$. 观测 $\mathrm{MX}_{2} / \mathrm{MX}_{2}$ 异质结构的层间激子跃迁能是 对 II 型半导体理论预测最直接的实验验证. 本课题 组 ${ }^{[35]}$ 利用 $\mathrm{PL}$ 光谱对转移到 $\mathrm{SiO}_{2} / \mathrm{Si}$ 衬底上的 $\mathrm{MoS}_{2} /$ $\mathrm{WS}_{2}$ 和 $\mathrm{WS}_{2} / \mathrm{MoS}_{2}$ 层间异质结构进行了系统的表征, 如图6(a)所示. 发现 $\mathrm{MoS}_{2}$ 和 $\mathrm{WS}_{2}$ 的PL特征峰分别位 于 1.86 和 $1.98 \mathrm{eV}$, 这两个特征峰的位置与前期报道
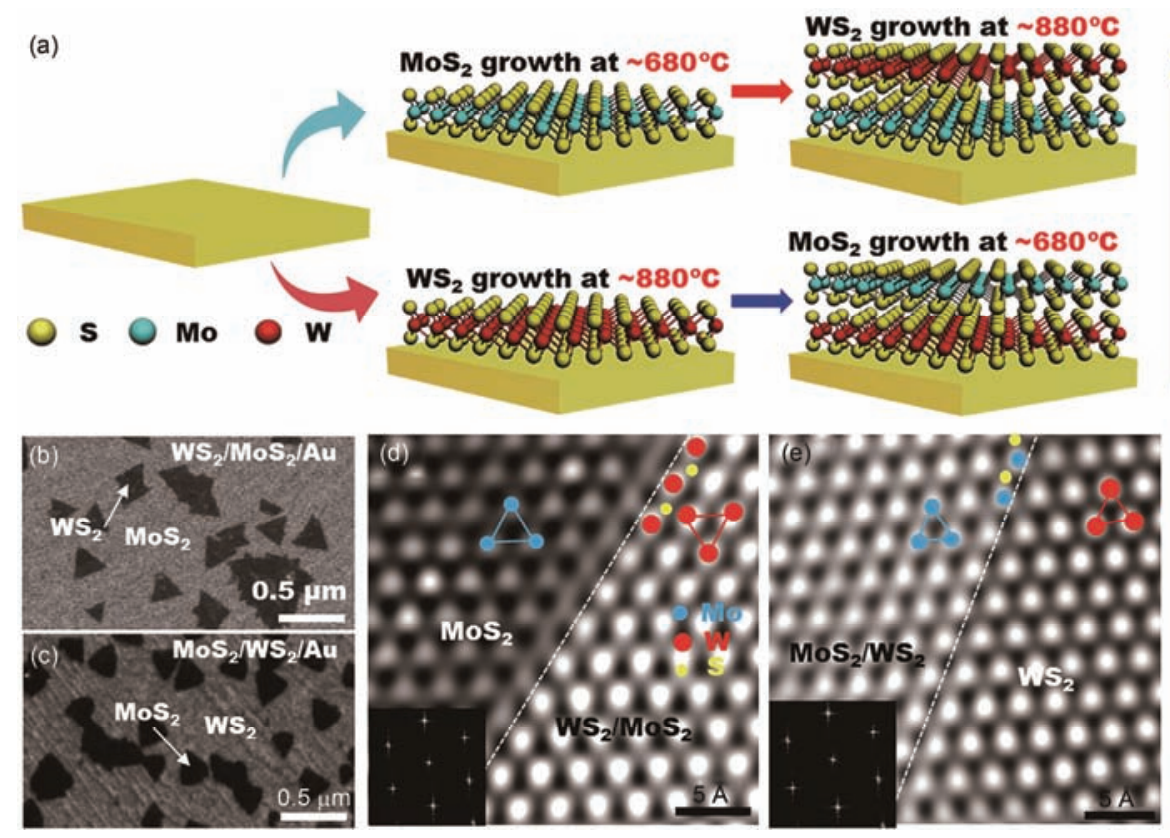
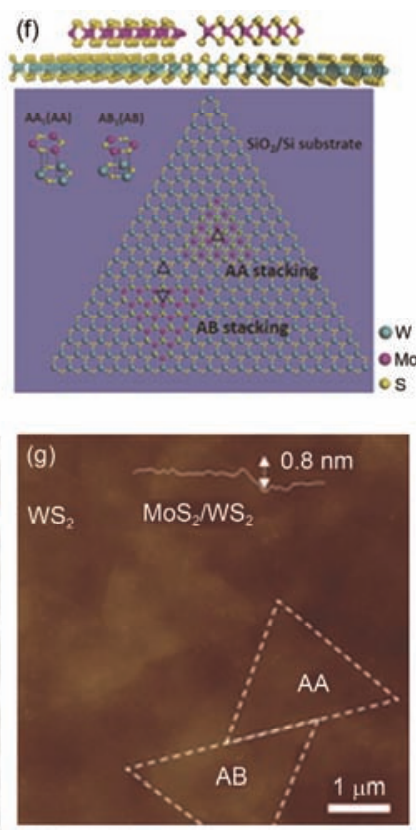

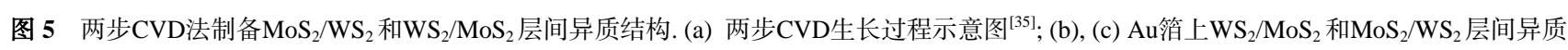
结构的SEM图 ${ }^{[35]}$; (d), (e) $\mathrm{WS}_{2} / \mathrm{MoS}_{2}$ 和 $\mathrm{MoS}_{2} / \mathrm{WS}_{2}$ 层间异质结构的STEM图 ${ }^{[35]}$; (f) 具有A-A和A-B堆垛的 $\mathrm{MoS}_{2} / \mathrm{WS}_{2}$ 层间异质结构的示意图 ${ }^{[64]}$; (g) $\mathrm{MoS}_{2} / \mathrm{WS}_{2}$ 层间异质结构的AFM图 ${ }^{[64]}$

Figure 5 Two-step CVD syntheses of $\mathrm{MoS}_{2} / \mathrm{WS}_{2}$ and $\mathrm{WS}_{2} / \mathrm{MoS}_{2}$ vertical heterostructures. (a) Schematic illustrations of the two-step CVD growth processes ${ }^{[35]}$; (b), (c) SEM images of $\mathrm{WS}_{2} / \mathrm{MoS}_{2}$ and $\mathrm{MoS}_{2} / \mathrm{WS}_{2}$ vertical heterostructures on Au foils ${ }^{[35]}$; (d), (e) STEM images of WS $/ \mathrm{MoS}_{2}$ and $\mathrm{MoS}_{2} / \mathrm{WS}_{2}$ vertical heterostructures ${ }^{[35]}$; (f) schematic illustration of vertically assembled A-A and A-B stacked $\mathrm{MoS}_{2} / \mathrm{WS}_{2}$ heterostructures on $\mathrm{SiO}_{2} / \mathrm{Si}^{[64]}$; (g) AFM image of $\mathrm{MoS}_{2} / \mathrm{WS}_{2}$ vertical heterostructure ${ }^{[64]}$ 

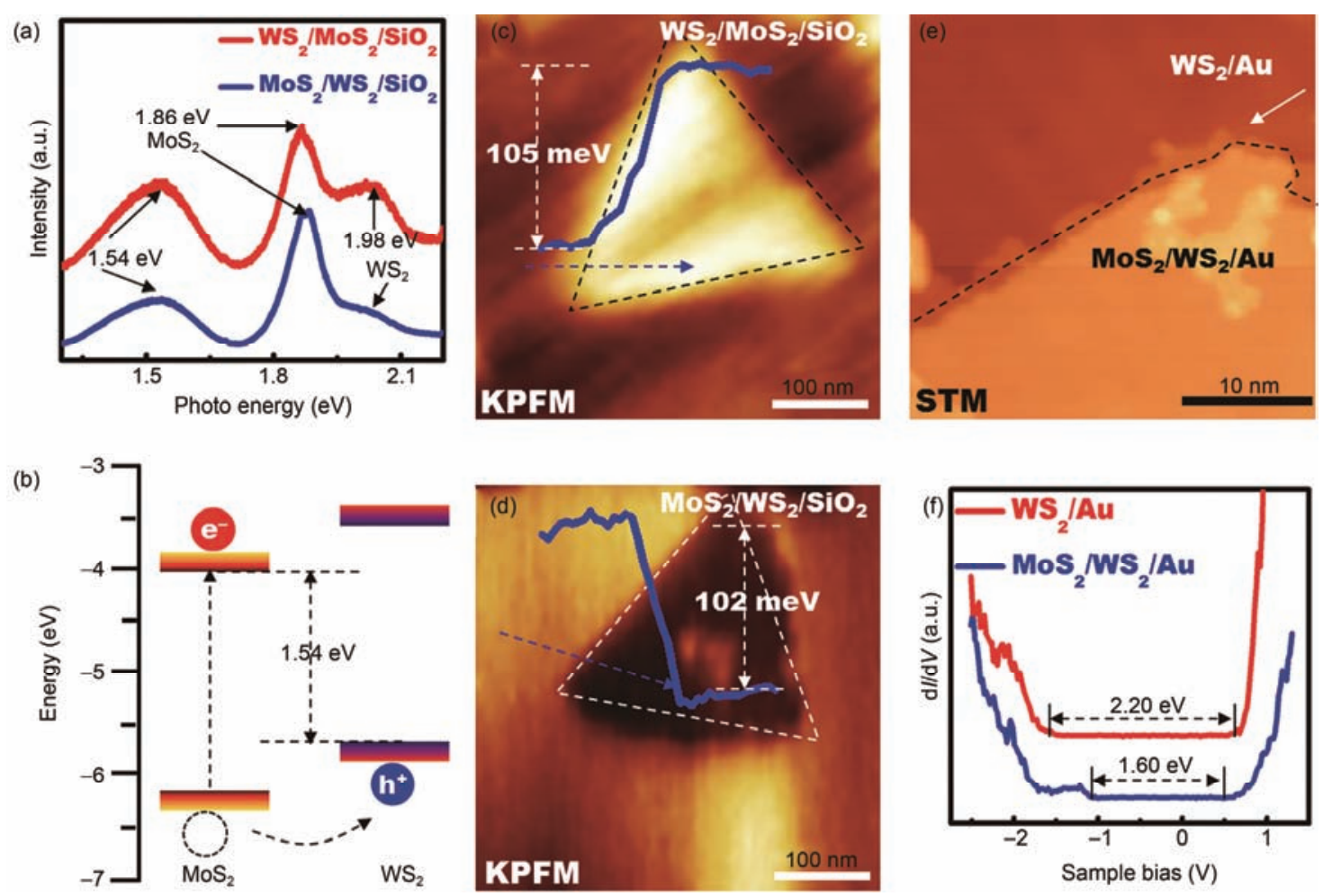

图 $6 \mathrm{MoS}_{2} / \mathrm{WS}_{2}$ 和 $\mathrm{WS}_{2} / \mathrm{MoS}_{2}$ 异质结构的层间激子跃迁能和局域电学性质. (a) $\mathrm{MoS}_{2} / \mathrm{WS}_{2}$ 和 $\mathrm{WS}_{2} / \mathrm{MoS}_{2}$ 层间异质结构的光致发光谱; (b)

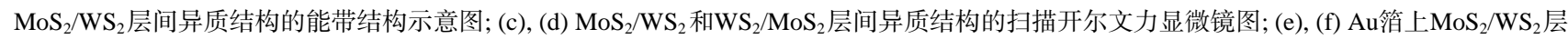
间异质结构的STM/STS(扫描条件: $V_{\mathrm{s}}=1.20 \mathrm{~V}, I_{\mathrm{t}}=0.20 \mathrm{nA}, T=78 \mathrm{~K}, 10 \mathrm{mV}, 932 \mathrm{~Hz}$ ) ${ }^{[35]}$

Figure 6 The interlayer excitonic transition energies and the local electrical properties of $\mathrm{MoS}_{2} / \mathrm{WS}_{2}$ and $\mathrm{WS}_{2} / \mathrm{MoS}_{2}$ vertical heterostructures. (a) PL spectra of $\mathrm{MoS}_{2} / \mathrm{WS}_{2}$ and $\mathrm{WS}_{2} / \mathrm{MoS}_{2}$ vertical heterostructures on $\mathrm{SiO}_{2} / \mathrm{Si}$ substrates; (b) schematic view of the band alignment of $\mathrm{MoS}_{2} / \mathrm{WS}_{2}$ vertical heterostructure; (c), (d) SKPFM images of $\mathrm{MoS}_{2} / \mathrm{WS}_{2}$ and $\mathrm{WS}_{2} / \mathrm{MoS}_{2}$ vertical heterostructures on $\mathrm{SiO}_{2} / \mathrm{Si}$ substrates; (e), (f) STM/STS characterizations of $\mathrm{MoS}_{2} / \mathrm{WS}_{2}$ vertical heterostructure on Au foils $\left(V_{\mathrm{s}}=1.20 \mathrm{~V}, I_{\mathrm{t}}=0.20 \mathrm{nA}, T=78 \mathrm{~K}, 10 \mathrm{mV}, 932 \mathrm{~Hz}\right)^{[35]}$

的绝缘基底上获得的单层 $\mathrm{MoS}_{2}{ }^{[21]}$ 和 $\mathrm{WS}_{2}{ }^{[23]}$ 一致, 这 表明, 利用两步 CVD法制备的 $\mathrm{MoS}_{2} / \mathrm{WS}_{2}$ 和 $\mathrm{WS}_{2} / \mathrm{MoS}_{2}$ 层间异质结构没有合金相和元素互混. 特别地, $\mathrm{MoS}_{2} / \mathrm{WS}_{2}$ 和 $\mathrm{WS}_{2} / \mathrm{MoS}_{2}$ 层间异质结构的 PL光谱中, 光子能量为 $1.54 \mathrm{eV}$ 的位置均存在一个明显的特征峰. 该特征峰只存在于异质结构区域, 在单一 $\mathrm{MoS}_{2}$ 和 $\mathrm{WS}_{2}$ 上则明显消失, 这表明该特征峰是 $\mathrm{MoS}_{2} / \mathrm{WS}_{2}$ 和 $\mathrm{WS}_{2} / \mathrm{MoS}_{2}$ 层间异质结构特有的. 需要指出的是, 该 特征峰的光子能量与理论计算 $\mathrm{MoS}_{2} / \mathrm{WS}_{2}$ 异质结构的 层间激子跃迁能完全一致(图6(b) $)^{[15]}$. 从而说明, 在 实验上直接观察到了 $\mathrm{MoS}_{2} / \mathrm{WS}_{2}$ 和 $\mathrm{WS}_{2} / \mathrm{MoS}_{2}$ 异质结 构的层间激子跃迁能. 以上实验结果表明, 利用两步 CVD方法获得了本征 $\mathrm{MoS}_{2} / \mathrm{WS}_{2}$ 和 $\mathrm{WS}_{2} / \mathrm{MoS}_{2}$ 层间异 质结构; $\mathrm{MoS}_{2}$ 与 $\mathrm{WS}_{2}$ 之间具有非常强的界面相互作 用; 异质界面处没有任何元素互混.

此外, $\mathrm{MoS}_{2} / \mathrm{WS}_{2}$ 和 $\mathrm{WS}_{2} / \mathrm{MoS}_{2}$ 层间异质结构的局 域电学性质是未来电子和光电子学应用的关键参数. 扫描开尔文力显微镜(scanning Kelvin probe force microscopy, SKPFM) 和 STM/STS 是进行 $\mathrm{MX}_{2} / \mathrm{MX}_{2}$ 异 质结构局域电学性质表征的有力手段. 利用 SKPFM 对转移到 $\mathrm{SiO}_{2} / \mathrm{Si}$ 衬底上的 $\mathrm{MoS}_{2} / \mathrm{WS}_{2}$ 和 $\mathrm{WS}_{2} / \mathrm{MoS}_{2}$ 层 间异质结构进行了系统研究. 图6(c), (d)显示了 $\mathrm{WS}_{2} /$ $\mathrm{MoS}_{2}$ 和 $\mathrm{MoS}_{2} / \mathrm{WS}_{2}$ 两种层间异质结构的表面电势分 布. 明显的亮暗衬度证明所获得 $\mathrm{MoS}_{2} / \mathrm{WS}_{2}$ 和 $\mathrm{WS}_{2} /$ $\mathrm{MoS}_{2}$ 是本征的异质结构而非合金相. 与此同时, 计 算了 $\mathrm{MoS}_{2}$ 和 $\mathrm{WS}_{2}$ 的费米能级差, 分别为 $105 \mathrm{meV}$ $\left(\mathrm{WS}_{2} / \mathrm{MoS}_{2}\right)$ 和 $102 \mathrm{meV}\left(\mathrm{MoS}_{2} / \mathrm{WS}_{2}\right)$, 这与前期报道的 $\mathrm{SiO}_{2} / \mathrm{Si}$ 衬底上两步 $\mathrm{CVD}$ 制备的 $\mathrm{WS}_{2}-\mathrm{MoS}_{2}$ 面内异质结 构一致 $(107 \mathrm{meV})^{[65]}$. 需要指出的是, 异质界面处费 米能级差会形成一个自 $\mathrm{WS}_{2}$ 层到 $\mathrm{MoS}_{2}$ 层的内建电场, 进而导致了电子的定向移动，这种电荷的再分布极 大地增强了光伏响应和发光效应, 有望在未来光电 器件和二维激光等领域得到应用. 另外, 利用低温 $\mathrm{STM} / \mathrm{STS}$ 在原子尺度对 $\mathrm{Au}$ 簿上 $\mathrm{MoS}_{2} / \mathrm{WS}_{2}$ 层间异质 
结构的微观形貌和局域电子结构进行了表征, 如图 6(e), (f) 所示. $\mathrm{Au}$ 箔上单层 $\mathrm{WS}_{2}$ 的带隙宽度为 $2.2 \mathrm{eV}$, 而 $\mathrm{MoS}_{2} / \mathrm{WS}_{2}$ 的带隙宽度则减小到 $1.6 \mathrm{eV}$. 异质结构 区域带隙宽度的明显减小再次证明 $\mathrm{MoS}_{2} / \mathrm{WS}_{2}$ 形成了 II 型能带分布. 这种 II 异质结的建立促进了电子-空 穴对的自发分离, 有望在光催化析氢反应中得到应用.

\section{3 $\mathrm{Au}$ 簿上 $\mathrm{MoS}_{2} / \mathrm{WS}_{2}$ 和 $\mathrm{WS}_{2} / \mathrm{MoS}_{2}$ 层间异质结构 在光催化析氢反应中的应用}

依据理论计算, $\mathrm{MoS}_{2} / \mathrm{WS}_{2}$ 层间异质结构中, 光 激化电子和空穴分别位于 $\mathrm{MoS}_{2}$ 和 $\mathrm{WS}_{2}$ 上, 这将极大 增强光催化析氢反应活性 ${ }^{[66]}$. 为此, 将 $\mathrm{MoS}_{2} / \mathrm{WS}_{2} / \mathrm{Au}$ 和 $\mathrm{WS}_{2} / \mathrm{MoS}_{2} / \mathrm{Au}$ 直接应用于光催化析氢反应, 作为 对比, 也研究了 $\mathrm{Au}$ 䈃, $\mathrm{MoS}_{2} / \mathrm{Au}$ 和 $\mathrm{WS}_{2} / \mathrm{Au}$ 的光催化特 性, 如图7(a)所示.

暗态下, 这 5 种样品的析氢效率(电流密度为 3
$\mathrm{mA} \mathrm{cm}{ }^{-2}$ 时, 对应的过电势)排序如下: $\mathrm{MoS}_{2} / \mathrm{WS}_{2} / \mathrm{Au}$ $(-0.192 \mathrm{~V})>\mathrm{WS}_{2} / \mathrm{MoS}_{2} / \mathrm{Au}(-0.252 \mathrm{~V})>\mathrm{WS}_{2} / \mathrm{Au}(-0.382$ $\mathrm{V})>\mathrm{MoS}_{2} / \mathrm{Au}(-0.408 \mathrm{~V})>\mathrm{Au}$ 䇴 $(-0.457 \mathrm{~V}) . \mathrm{MoS}_{2} / \mathrm{WS}_{2} /$ $\mathrm{Au}$ 和 $\mathrm{WS}_{2} / \mathrm{MoS}_{2} / \mathrm{Au}$ 增强的析氢反应活性起源于这两 类层间异质结构的 II 型能带分布. 特别地, 对于 $\mathrm{WS}_{2} / \mathrm{MoS}_{2}$ 层间异质结构, 由于单层 $\mathrm{WS}_{2}$ 的导带高于 单层 $\mathrm{MoS}_{2}$, 因此来自电极的电子经由 $\mathrm{MoS}_{2}$ 注人 $\mathrm{WS}_{2}$ 时需要克服一定的势垒, 这将极大地减弱 $\mathrm{WS}_{2} / \mathrm{MoS}_{2}$ 的析氢效率; 对于 $\mathrm{MoS}_{2} / \mathrm{WS}_{2}$ 层间异质结构, II 型能 带分布促进了电子由 $\mathrm{WS}_{2}$ 向 $\mathrm{MoS}_{2}$ 的传输, 进而增强 了 $\mathrm{MoS}_{2} / \mathrm{WS}_{2}$ 析氢反应活性. 令人惊奇的是, 在模拟 太阳光的辐照下, $\mathrm{MoS}_{2} / \mathrm{WS}_{2} / \mathrm{Au}$ 的光催化析氢反应效 率极大增强, 而 $\mathrm{WS}_{2} / \mathrm{MoS}_{2} / \mathrm{Au}$ 的析氢效率则明显减 弱(图7(c)). 图7(d)阐述了 $\mathrm{MoS}_{2} / \mathrm{WS}_{2} / \mathrm{Au}$ 电子传输机 制，来自 $\mathrm{WS}_{2}$ 的光激化电子注人到了 $\mathrm{MoS}_{2}$ 层，并还 原了电解液中的氢离子, 产生氢气, 残留在 $\mathrm{WS}_{2}$ 中的
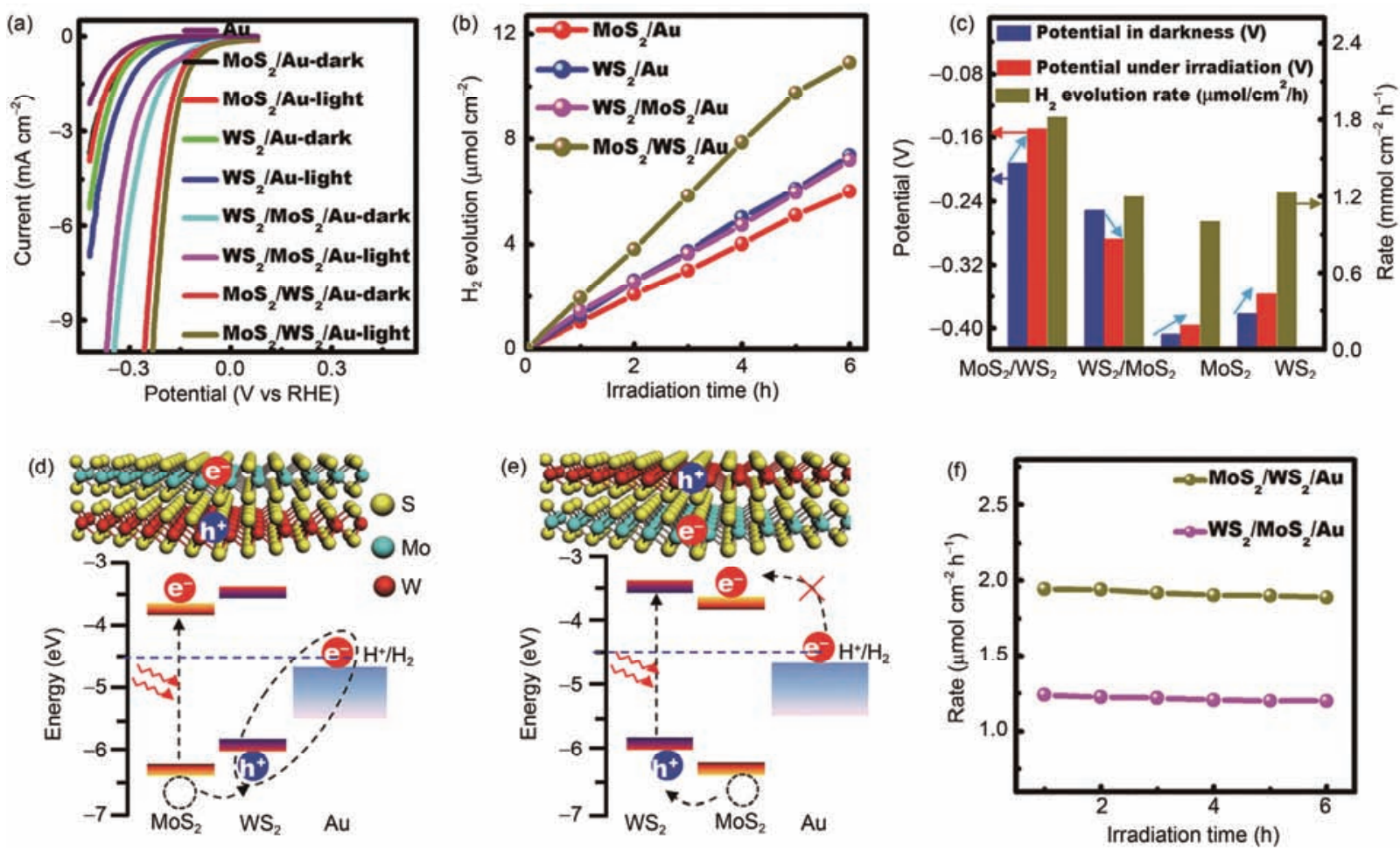

图 $7 \mathrm{MoS}_{2} / \mathrm{WS}_{2}$ 和 $\mathrm{WS}_{2} / \mathrm{MoS}_{2}$ 层间异质结构在光催化析氢反应中的应用. (a) $\mathrm{Au}$ 簿, $\mathrm{MoS}_{2} / \mathrm{Au}, \mathrm{WS}_{2} / \mathrm{Au}, \mathrm{WS}_{2} / \mathrm{MoS}_{2} / \mathrm{Au}$ 和MoS $2 / \mathrm{WS}_{2} / \mathrm{Au}$ 的线性扫描 伏安曲线; (b) $\mathrm{MoS}_{2} / \mathrm{Au}, \mathrm{WS}_{2} / \mathrm{Au}, \mathrm{WS}_{2} / \mathrm{MoS}_{2} / \mathrm{Au}$ 和MoS $2 / \mathrm{WS}_{2} / \mathrm{Au}$ 的光催化析氢曲线; (c) $\mathrm{MoS}_{2} / \mathrm{Au}, \mathrm{WS}_{2} / \mathrm{Au}, \mathrm{WS}_{2} / \mathrm{MoS}_{2} / \mathrm{Au}$ 和MoS $2 / \mathrm{WS}_{2} / \mathrm{Au}$ 的产氢 速率和过电势; (d), (e) $\mathrm{MoS}_{2} / \mathrm{WS}_{2} / \mathrm{Au}$ 和WS $/ \mathrm{MoS}_{2} / \mathrm{Au}$ 的电子传输机制示意图; (f) $\mathrm{WS}_{2} / \mathrm{MoS}_{2} / \mathrm{Au}$ 和MoS $/ \mathrm{WS}_{2} / \mathrm{Au}$ 的产氢速率与辐照时间关系图 ${ }^{[35]}$ Figure 7 The applications of $\mathrm{MoS}_{2} / \mathrm{WS}_{2}$ and $\mathrm{WS}_{2} / \mathrm{MoS}_{2}$ vertical heterostructures in photocatalytic hydrogen evolution reaction. (a) Linear sweep voltammetry curves of $\mathrm{Au}$ foil, $\mathrm{MoS}_{2} / \mathrm{Au}, \mathrm{WS}_{2} / \mathrm{Au}, \mathrm{WS}_{2} / \mathrm{MoS}_{2} / \mathrm{Au}$ and $\mathrm{MoS}_{2} / \mathrm{WS}_{2} / \mathrm{Au}$, respectively; (b) photocatalytic $\mathrm{H}_{2}$ evolution curves of MoS $/ \mathrm{Au}$, $\mathrm{WS}_{2} / \mathrm{Au}, \mathrm{WS}_{2} / \mathrm{MoS}_{2} / \mathrm{Au}$ and $\mathrm{MoS}_{2} / \mathrm{WS}_{2} / \mathrm{Au}$, respectively; (c) the $\mathrm{H}_{2}$ evolution rates and the potentials of $\mathrm{MoS}_{2} / \mathrm{Au}, \mathrm{WS}_{2} / \mathrm{Au}, \mathrm{WS}_{2} / \mathrm{MoS}{ }_{2} / \mathrm{Au}$ and $\mathrm{MoS}_{2} / \mathrm{WS}_{2} / \mathrm{Au}$ respectively; (d), (e) schematic illustrations of the electron transfer mechanisms in $\mathrm{WS}_{2} / \mathrm{MoS}_{2}$ and $\mathrm{MoS}_{2} / \mathrm{WS}_{2}$ vertical heterostructures; (f) irradiation time dependence of the $\mathrm{H}_{2}$ evolution rate for $\mathrm{WS}_{2} / \mathrm{MoS}_{2}$ and $\mathrm{MoS}_{2} / \mathrm{WS}_{2}$ vertical heterostructures ${ }^{[35]}$ 
光激化空穴则被来自电极中的电子中和, 这种光激 化电子和空穴对的有效分离促进了光催化析氢反应 的进行. 此外, 对 $\mathrm{WS}_{2} / \mathrm{MoS}_{2} / \mathrm{Au}$ 光激化电子传输机制 进行了分析(图7(e)). $\mathrm{WS}_{2}$ 层中的光激化电子传输到 了 $\mathrm{MoS}_{2}$ 层, 从而减小了 $\mathrm{WS}_{2} / \mathrm{MoS}_{2} / \mathrm{Au}$ 表面层的电子 密度. 富集在 $\mathrm{MoS}_{2}$ 层的光激化电子也将阻碍来自电 极的电子经由 $\mathrm{MoS}_{2}$ 传输到 $\mathrm{WS}_{2} / \mathrm{MoS}_{2} / \mathrm{Au}$ 表面层. 这 两个因素协同作用导致光照下 $\mathrm{WS}_{2} / \mathrm{MoS}_{2} / \mathrm{Au}$ 的光催 化析氢反应效率极大降低. 与此同时, 计算了 $\mathrm{MoS}_{2} /$ $\mathrm{Au}, \mathrm{WS}_{2} / \mathrm{Au}, \mathrm{WS}_{2} / \mathrm{MoS}_{2} / \mathrm{Au}$ 和 $\mathrm{MoS}_{2} / \mathrm{WS}_{2} / \mathrm{Au}$ 的光催化 析氢速率, 分别为 $1.003,1.230,1.201$ 和 $1.819 \mu \mathrm{mol}$ $\mathrm{cm}^{-2} \mathrm{~h}^{-1}$ (图7(b)). $\mathrm{MoS}_{2} / \mathrm{WS}_{2} / \mathrm{Au}$ 和 $\mathrm{WS}_{2} / \mathrm{MoS}_{2} / \mathrm{Au}$ 的光 催化析氢反应速率与光辐照时间关系表明, 这两种 异质结构均具有非常高效的催化稳定性, 如图7(f)所 示. 以上光催化析氢反应结果表明, $\mathrm{MoS}_{2} / \mathrm{WS}_{2}$ 层间 异质结构, 因其独特的 II 型能带分布, 促进了电子的 定向流动, 是一种潜在的光催化析氢反应催化剂.

总之, 利用两步 CVD方法在 $A u$ 䇴上选择性的制 备了 $\mathrm{MoS}_{2} / \mathrm{WS}_{2}$ 和 $\mathrm{WS}_{2} / \mathrm{MoS}_{2}$ 层间异质结构. 因两步 CVD方法的选择, 有效避免了 Mo和W两种元素的互 混, 从而获得了本征 $\mathrm{MoS}_{2} / \mathrm{WS}_{2}$ 和 $\mathrm{WS}_{2} / \mathrm{MoS}_{2}$ 层间异质 结构. 利用PL光谱直接观测到了这两种异质结构的 层间激子跃迁能, 表明 II 型能带结构的直接构筑. 此 外, $\mathrm{MoS}_{2} / \mathrm{WS}_{2}$ 和 $\mathrm{WS}_{2} / \mathrm{MoS}_{2}$ 层间异质结构也展现出了 截然相反的光催化析氢反应特性.

\section{5 结论与展望}

$\mathrm{MX}_{2} /$ garphene层间异质结构利用了单层 $\mathrm{MX}_{2}$ 直 接带隙的能带结构和 graphene超高的电子传输特性, 在电子、光电子、电催化析氢反应和太阳能电池等领 域具有非常广泛的应用前景. 大畴区、大面积、可控 制备高质量 $\mathrm{MX}_{2} /$ graphene层间异质结构是实现这些 应用的先决条件. 不同国家和地区的研究组纷纷加 人到了 $\mathrm{MX}_{2} /$ garphene层间异质结构的制备领域中来, 机械剥离/堆垛、 $\left(\mathrm{NH}_{4}\right)_{2} \mathrm{MX}_{4}$ 盐溶液的热分解、分子束
外延生长、金属有机化学气相沉积、CVD等方法纷 纷呈现. 本课题组利用两步 CVD方法实现了 $\mathrm{Au}$ 䇴上 $\mathrm{MoS}_{2} /$ graphene层间异质结构的可控制备, 利用低温 $\mathrm{STM} / \mathrm{STS}$ 获得了 $\mathrm{MoS}_{2} /$ graphene层间异质结构的原子 尺度形貌和局域电子结构, 为理解 CVD异质结构的 晶体质量、能带结构和器件性能提供了最直接的实验 依据, 实现了 $\mathrm{MoS}_{2} /$ graphene层间异质结构在电催化 析氢反应中的高效应用.

$\mathrm{MX}_{2} / \mathrm{MX}_{2}$ 层间异质结构是一种 II 型半导体, 导 带和价带分别位于两种 $\mathrm{MX}_{2}$ 材料上, 有利于光激化 电子-空穴对的有效分离, 在光伏器件、二维激光器 和光催化析氢反应等领域具有广阔的应用前景. 美 国、英国、德国、韩国、西班牙、日本等国家和中国 科学院物理研究所、国家纳米科学研究中心、北京大 学、清华大学、南京大学、湖南大学的相关课题组相 继开展了研究, 使 $\mathrm{MX}_{2} / \mathrm{MX}_{2}$ 层间异质结构的制备、新 奇物理性质研究和应用探索进人了一个稳定的较快 发展时期, 并正在形成一个新的研究热点和领域. 本 课题组利用两步 CVD的方法在 $\mathrm{Au}$ 箔上选择性地制备 了 $\mathrm{MoS}_{2} / \mathrm{WS}_{2}$ 和 $\mathrm{WS}_{2} / \mathrm{MoS}_{2}$ 层间异质结构; 直接观察到 了这两种异质结构的层间激子跃迁能, 为理论预测 的 II 型半导体提供了实验依据; 将 $\mathrm{Au}$ 管上制备的 $\mathrm{MoS}_{2} / \mathrm{WS}_{2}$ 和 $\mathrm{WS}_{2} / \mathrm{MoS}_{2}$ 层间异质结构直接应用到光 催化析氢反应上, 并获得了截然相反的析氢效果.

尽管 $\mathrm{MX}_{2} /$ graphene和 $\mathrm{MX}_{2} / \mathrm{MX}_{2}$ 层间异质结构的 研究不断推陈出新, 然而所得异质结构畴区尺寸小、 层厚/扭转角不可控、界面易污染、不同元素易互混、 制备成本较高等现象仍然得不到根本解决. 因此, 实 现 $\mathrm{MX}_{2} /$ graphene和 $\mathrm{MX}_{2} / \mathrm{MX}_{2}$ 层间异质结构的批量制 备和实际应用需要集中各个领域的研究者进行联合 攻关. 但是必须要说明的是, 虽然要实现 $\mathrm{MX}_{2} / \mathrm{gra}$ phene和 $\mathrm{MX}_{2} / \mathrm{MX}_{2}$ 层间异质结构的大规模应用还有很 长一段距离, 但是这两类异质结构表现出来的优异 电学、光学、催化性质注定其在未来的器件和能源领 域中会发挥巨大作用.

\section{参考文献}

1 Wang Q, Kalantar-Zadeh K, Kis A, et al. Electronics and optoelectronics of two-dimensional transition metal dichalcogenides. Nat Nanotechnol, 2012, 7: 699-712

2 Radisavljevic B, Radenovic A, Brivio J, et al. Single-layer $\mathrm{MoS}_{2}$ transistors. Nat Nanotechnol, 2011, 6: 147-150

3 Zeng H, Dai J, Yao W. Valley polarization in $\mathrm{MoS}_{2}$ monolayers by optical pumping. Nat Nanotechnol, 2012, 7: 490-493

4 Yan R S, Simpson J R, Bertolazzi S, et al. Thermal conductivity of monolayer molybdenum disulfide obtained from temperature- 
dependent Raman spectroscopy. ACS Nano, 2014, 8: 986-993

5 Bertolazzi S, Brivio J, Kis A. Stretching and breaking of ultrathin $\mathrm{MoS}_{2}$. ACS Nano, 2011, 5: 9703-9709

6 Kang K, Xie S, Huang L, et al. High-mobility three-atom-thick semiconducting films with wafer-scale homogeneity. Nature, 2015, 520: 656-660

7 Lu J M, Zheliuk O, Leermakers I, et al. Evidence for two-dimensional Ising superconductivity in gated MoS 2 . Science, 2015, 350: 1353-1357

8 Yin X, Te Z, Chenet D A, et al. Edge nonlinear optics on a $\mathrm{MoS}_{2}$ atomic monolayer. Science, 2014, 344: 488-490

9 Mak K F, He K, Shan J, et al. Control of valley polarization in monolayer MoS 2 by optical helicity. Nat Nanotechnol, 2012, 7: 494-498

10 Du X, Skachko I, Barker A, et al. Approaching ballistic transport in suspended graphene. Nat Nanotechnol, 2008, 3: 491-495

11 Georgiou T, Jalil R, Belle B D, et al. Vertical field-effect transistor based on graphene- $\mathrm{WS}_{2}$ heterostructures for flexible and transparent electronics. Nat Nanotechnol, 2013, 8: 100-103

12 Yang J, Voiry D, Ahn S J, et al. Two-dimensional hybrid nanosheets of tungsten disulfide and reduced graphene oxide as catalysts for enhanced hydrogen evolution. Angew Chem Int Ed, 2013, 52: 13751-13754

13 Wang H, Bang J, Sun Y Y, et al. The role of collective motion in the ultrafast charge transfer in van der Waals heterostructures. Nat Commun, 2016, 7: 11504

14 Rivera P, Seyler K L, Yu H Y, et al. Valley-polarized exciton dynamics in a 2D semiconductor heterostructure. Science, 2016, 351: 688-691

15 Kośmider K, Fernández-Rossier J. Electronic properties of the $\mathrm{MoS}_{2}-\mathrm{WS}_{2}$ heterojunction. Phys Rev B, 2013, 87: 075451

16 Reina A, Jia X, Ho J, et al. Large area, few-layer graphene films on arbitrary substrates by chemical vapor deposition. Nano Lett, 2008, 9: $30-35$

17 Li X S, Cai W W, An J, et al. Large-area synthesis of high-quality and uniform graphene films on copper foils. Science, 2009, 324: 1312-1314

18 Shi Y, Hamsen C, Jia X, et al. Synthesis of few-layer hexagonal boron nitride thin film by chemical vapor deposition. Nano Lett, 2010, 10: 4134-4139

19 Kim K K, Hsu A, Jia X, et al. Synthesis of monolayer hexagonal boron nitride on Cu foil using chemical vapor deposition. Nano Lett, 2011, 12: 161-166

20 Lee Y H, Zhang X Q, Zhang W, et al. Synthesis of large-area $\mathrm{MoS}_{2}$ atomic layers with chemical vapor deposition. Adv Mater, 2012, 24: 2320-2325

21 Najmaei S, Liu Z, Zhou W, et al. Vapour phase growth and grain boundary structure of molybdenum disulphide atomic layers. Nat Mater, 2013, 12: 754-759

22 van der Zande A M, Huang P Y, Chenet D A, et al. Grains and grain boundaries in highly crystalline monolayer molybdenum disulphide. Nat Mater, 2013, 12: 554-561

23 Zhang Y, Zhang Y F, Ji Q Q, et al. Controlled growth of high-quality monolayer $\mathrm{WS}_{2}$ layers on sapphire and imaging its grain boundary. ACS Nano, 2013, 7: 8963-8971

24 Zhang Y, Ji Q Q, Han G F, et al. Dendritic, transferable, strictly monolayer $\mathrm{MoS}_{2}$ flakes synthesized on $\mathrm{SrTiO}_{3}$ single crystals for efficient electrocatalytic applications. ACS Nano, 2014, 8: 8617-8624

25 Shi J P, Ma D L, Han G F, et al. Controllable growth and transfer of monolayer $\mathrm{MoS}_{2}$ on Au foils and its potential application in hydrogen evolution reaction. ACS Nano, 2014, 8: 10196-10204

26 Shi J P, Yang Y, Zhang Y, et al. Monolayer $\mathrm{MoS}_{2}$ growth on Au foils and on-site domain boundary imaging. Adv Funct Mater, 2015, 25: 842-849

27 Shi J P, Zhang X N, Ma D L, et al. Substrate facet effect on the growth of monolayer $\mathrm{MoS}_{2}$ on Au foils. ACS Nano, 2015, 9: 4017-4025

28 Shi Y M, Zhou W, Lu Y A, et al. van der Waals epitaxy of $\mathrm{MoS}_{2}$ layers using graphene as growth templates. Nano Lett, 2012, 12: 2784-2791

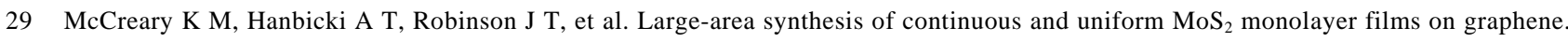
Adv Funct Mater, 2014, 24: 6449-6454

30 Gong Y J, Lin J H, Wang X L, et al. Vertical and in-plane heterostructures from $\mathrm{WS}_{2} / \mathrm{MoS}_{2}$ monolayers. Nat Mater, 2014, 13: 1135-1142

31 Zhang Q, Xiao X, Zhao R Q, et al. Two-dimensional layered heterostructures synthesized from core-shell nanowires. Angew Chem Int Ed, 2015, 54: 8957-8960

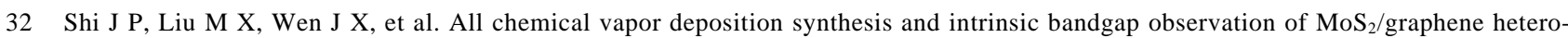
structures. Adv Mater, 2015, 27: 7086-7092

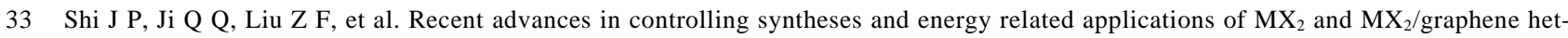
erostructures. Adv Energy Mater, 2016, 6: 1600459

34 Shi J P, Zhou X B, Han G F, et al. Narrow-gap quantum wires arising from the edges of monolayer MoS 2 synthesized on graphene. Adv Mater Interfaces, 2016, 3: 1600332

35 Shi J P, Tong R, Zhou X B, et al. Temperature-mediated selective growth of $\mathrm{MoS}_{2} / \mathrm{WS}_{2}$ and $\mathrm{WS}_{2} / \mathrm{MoS}_{2}$ vertical stacks on Au foils for direct photocatalytic applications. Adv Mater, 2016, 28: 10664-10672 
41 Yang J, Wang D, Han H, et al. Roles of cocatalysts in photocatalysis and photoelectrocatalysis. Acc Chem Res, 2013, 46: 1900-1909

42 Liao T, Sun Z Q, Sun C H, et al. Electronic coupling and catalytic effect on $\mathrm{H}_{2}$ evolution of MoS $2 /$ graphene nanocatalyst. Sci Rep, 2014, 4: 6256

43 Li Y G, Wang H L, Xie L M, et al. $\mathrm{MoS}_{2}$ Nanoparticles grown on graphene: An advanced catalyst for the hydrogen evolution reaction. J Am Chem Soc, 2011, 133: 7296-7299

44 Britnell L, Ribeiro R M, Eckmann A, et al. Strong light-matter interactions in heterostructures of atomically thin films. Science, 2013, 340: 1311-1314

45 Kaasbjerg K, Thygesen K S, Jacobsen K W. Phonon-limited mobility in n-type single-layer MoS 2 from first principles. Phys Rev B, 2012, 85: 115317

46 Allain A, Kang J H, Banerjee K, et al. Electrical contacts to two-dimensional semiconductors. Nat Mater, 2015, 14: 1195-1205

47 Cui X, Lee G H, Kim Y D, et al. Multi-terminal transport measurements of MoS $_{2}$ using a van der Waals heterostructure device platform. Nat Nanotechnol, 2015, 10: 534-540

48 Yu W J, Liu Y, Zhou H L, et al. Highly efficient gate-tunable photocurrent generation in vertical heterostructures of layered materials. Nat Nanotechnol, 2013, 8: 952-958

49 Ugeda M M, Bradley A J, Shi S F, et al. Giant bandgap renormalization and excitonic effects in a monolayer transition metal dichalcogenide semiconductor. Nat Mater, 2014, 13: 1091-1095

50 Fu L, Sun Y Y, Wu N, et al. Direct Growth of $\mathrm{MoS}_{2} / \mathrm{h}-\mathrm{BN}$ heterostructures via a sulfide-resistant alloy. ACS Nano, 2016, 10: 2063-2070

51 Gutiérrez H R, Perea-López N, Elías A L, et al. Extraordinary room-temperature photoluminescence in triangular WS 2 monolayers. Nano Lett, 2013, 13: 3447-3454

52 Hinnemann B, Moses P G, Bonde J, et al. Biomimetic hydrogen evolution: MoS $_{2}$ nanoparticles as catalyst for hydrogen evolution. J Am Chem Soc, 2005, 127: 5308-5309

53 Fang H, Battaglia C, Carraro C, et al. Strong interlayer coupling in van der Waals heterostructures built from single-layer chalcogenides. Proc Natl Acad Sci USA, 2014, 111: 6198-6202

54 Kozawa D, Carvalho A, Verzhbitskiy I, et al. Evidence for fast interlayer energy transfer in $\mathrm{MoSe}_{2} / \mathrm{WS}_{2}$ heterostructures. Nano Lett, 2016, 16: 4087-4093

55 Li M Y, Shi Y M, Cheng C C, et al. Epitaxial growth of a monolayer $\mathrm{WSe}_{2}-\mathrm{MoS}_{2}$ lateral p-n junction with an atomically sharp interface. Science, 2015, 349: 524-528

56 Duan X D, Wang C, Shaw J C, et al. Lateral epitaxial growth of two-dimensional layered semiconductor heterojunctions. Nat Nanotechnol, 2014, 9: 1024-1030

57 Son Y, Li M Y, Cheng C C, et al. Observation of switchable photoresponse of a monolayer $\mathrm{WSe}_{2}-\mathrm{MoS}_{2}$ lateral heterostructure via photocurrent spectral atomic force microscopic imaging. Nano Lett, 2016, 16: 3571-3577

58 Chen K, Wan X, Wen J X, et al. Electronic properties of $\mathrm{MoS}_{2}-\mathrm{WS}_{2}$ heterostructures synthesized with two-step lateral epitaxial strategy. ACS Nano, 2015, 9: 9868-9876

59 Hong X P, Kim J, Shi S F, et al. Ultrafast charge transfer in atomically thin $\mathrm{MoS}_{2} / \mathrm{WS}_{2}$ heterostructures. Nat Nanotechnol, 2014, 9: 682-686

60 Rivera P, Schaibley J R, Jones A M, et al. Observation of long-lived interlayer excitons in monolayer MoSe -WSe $_{2}$ heterostructures. Nat Commun, 2015, 6: 6242

61 Lin Y C, Ghosh R K, Addou R, et al. Atomically thin resonant tunnel diodes built from synthetic van der Waals heterostructures. Nat Commun, 2015, 6: 7311

62 Tongay S, Fan W, Kang J, et al. Tuning interlayer coupling in large-area heterostructures with CVD grown MoS and $_{2} \mathrm{~S}_{2}$ monolayers. Nano Lett, 2014, 14: 3185-3190

63 Gong Y J, Lei S D, Ye G L, et al. Two-step growth of two-dimensional WSe 2 /MoSe 2 heterostructures. Nano Lett, 2015, 15: 6135-6141

64 Zhang J, Wang J H, Chen P, et al. Observation of strong interlayer coupling in $\mathrm{MoS}_{2} / \mathrm{WS}_{2}$ heterostructures. Adv Mater, 2016, 28: $1950-1956$

65 Chen K, Wan X, Xie W, et al. Lateral built-in potential of monolayer $\mathrm{MoS}_{2}-\mathrm{WS}_{2}$ in-plane heterostructures by a shortcut growth strategy. Adv Mater, 2015, 27: 6431-6437

66 Kang J, Tongay S, Zhou J, et al. Band offsets and heterostructures of two-dimensional semiconductors. Appl Phys Lett, 2013, 102: 012111 


\title{
Controllable syntheses and energy related applications of $\mathrm{MX}_{2}$ heterostructures
}

\author{
SHI JianPing ${ }^{1,2}$, ZHOU XieBo ${ }^{1,2}$, ZHANG ZhePeng $^{2} \&$ ZHANG YanFeng ${ }^{1,2^{*}}$ \\ ${ }^{1}$ Department of Materials Science and Engineering, College of Engineering, Peking University, Beijing 100871, China; \\ ${ }^{2}$ Center for Nanochemistry (CNC), College of Chemistry and Molecular Engineering, Peking University, Beijing 100871, China \\ * Corresponding author, E-mail: yanfengzhang@pku.edu.cn
}

Two-dimensional (2D) layered materials have been a central focus of materials research since the discovery of graphene. Each layer in 2D layered materials consists of a covalently bonded and is weakly bound to neighboring layers by van der Waals interactions. This makes it feasible to mix and match highly disparate atomic layers to create a wide range of vertical heterostructures without the constraints of lattice matching and processing compatibility. In these heterostructures, different physical and chemical properties accompany with the stacked materials are combined, furthermore, many applications in electronic/optoelectronic devices and renewable energy storage/conversions are demonstrated. Notably, the controllable syntheses of large area, large domain size and intrinsic vertical heterostructures are the first step. In this review, recent research achievements towards the controllable syntheses and novel physical properties of $\mathrm{MX}_{2} /$ graphene and $\mathrm{MX}_{2} / \mathrm{MX}_{2}$ vertical heterostructures, as well as their applications in electrocatalytic/photocatalytic hydrogen evolution reaction are highlighted.

Firstly, by using a facile chemical vapor deposition (CVD) approach, high quality and coverage-tunable monolayer $\mathrm{MoS}_{2}$ /graphene vertical heterostructures on conventional $\mathrm{Au}$ foil substrates are demonstrated. The unique system is selected in view of the tunability of the coverage of $\mathrm{MoS}_{2}$, or the densities of active sites in hydrogen evolution reaction (HER), as well as its compatibility with scanning tunneling microscopy/spectroscopy (STM/STS) observations. Spectroscopic characterizations reveal a quasi-freestanding monolayer $\mathrm{MoS}_{2}$ feature, which is evidenced by a very weak n-doping effect and an intrinsic bandgap of $\mathrm{MoS}_{2}$ in the $\mathrm{MoS}_{2} /$ graphene/Au sandwich, as obtained from STM/STS characterizations. Moreover, the exciton binding energy is also deduced by combining photoluminescence measurements. For monolayer $\mathrm{MoS}_{2}$ synthesized on graphene/Au foils, a dramatic decrease of the bandgap from 2.20 to $0.30 \mathrm{eV}$ occurs at the domain edge within a lateral distance of $6 \mathrm{~nm}$, as evidenced by STM/STS observations. The edges of monolayer $\mathrm{MoS}_{2}$ triangles on graphene/Au foils can thus be regarded as narrow-gap quantum wires considering of their reduced bandgaps. More intriguingly, the bandgap decrease at the domain edge is closely related to the rather high HER performance for $\mathrm{MoS}_{2} /$ graphene/Au foils comparing with that of $\mathrm{MoS}_{2} / \mathrm{Au}$ foils.

Secondly, a growth-temperature-mediated two-step CVD strategy is designed to synthesize either $\mathrm{MoS}_{2} / \mathrm{WS}_{2}$ or $\mathrm{WS}_{2} / \mathrm{MoS}_{2}$ vertical heterostructures on Au foils in a controllable manner. The Au foil substrate is selected in view of its concurrently compatible with the growth of $\mathrm{MoS}_{2}$ and $\mathrm{WS}_{2}$ domains and even complete layers at considerably different temperatures. This unique growth system is also suitable for on-site STM/STS observations and direct photocatalysis applications. A dominant A-A stacked $\mathrm{MoS}_{2} / \mathrm{WS}_{2}$ and A-B stacked $\mathrm{WS}_{2} / \mathrm{MoS}_{2}$ heterostructures are selectively achieved and convinced by spherical-aberration corrected scanning transmission electron microscope (STEM) characterizations. The quasiparticle bandgap of $\mathrm{MoS}_{2} / \mathrm{WS}_{2} / \mathrm{Au}$ foils is unambiguously determined as $1.60 \pm 0.08 \mathrm{eV}$ by STM/STS. More intriguingly, the as-grown $\mathrm{MoS}_{2} / \mathrm{WS}_{2}$ stacks is found to possess higher photocatalytic activity in HER than that of $\mathrm{MoS}_{2}$, $\mathrm{WS}_{2}$, and $\mathrm{WS}_{2} / \mathrm{MoS}_{2}$, due to the effective electron-hole separation and the fast electron transfer kinetics. Motivated by those observations, we believe that significant research interests would be sparked about the oriented syntheses and the versatile applications of $\mathrm{MX}_{2}$-based heterostructures.

Finally, remaining challenges for the controllable syntheses and the large-scale applications of $\mathrm{MX}_{2} /$ graphene and $\mathrm{MX}_{2} / \mathrm{MX}_{2}$ vertical heterostructures are discussed, and the future research directions in the related fields are also proposed.

graphene, transition metal dichalcogenides, vertical heterostructures, electrocatalytic hydrogen evolution reaction, photocatalytic hydrogen evolution reaction

doi: 10.1360/N972016-01387 